\title{
SimCP: A Simulation Platform to Predict Gait Performance Following Orthopedic Intervention in Children With Cerebral Palsy
}

\begin{abstract}
Lorenzo Pitto ${ }^{1 *}$, Hans Kainz ${ }^{1}$, Antoine Falisse ${ }^{1}$, Mariska Wesseling ${ }^{1}$, Sam Van Rossom ${ }^{1}$, Hoa Hoang ${ }^{1}$, Eirini Papageorgiou ${ }^{2,3}$, Ann Hallemans ${ }^{4}$, Kaat Desloovere ${ }^{2,3}$, Guy Molenaers ${ }^{5,6}$, Anja Van Campenhout ${ }^{5,6}$, Friedl De Groote ${ }^{1}$ and IIse Jonkers ${ }^{1}$

${ }^{1}$ Department of Movement Sciences, KU Leuven, Leuven, Belgium, ${ }^{2}$ Department of Rehabilitation Sciences, Doctoral School of Biomedical Sciences, KU Leuven, Leuven, Belgium, ${ }^{3}$ Clinical Motion Analysis Laboratory, University Hospitals Leuven, Leuven, Belgium, ${ }^{4}$ Department of Rehabilitation Sciences and Physiotherapy, University of Antwerp, Antwerp, Belgium, ${ }^{5}$ Department of Orthopedics, University Hospitals Leuven, Leuven, Belgium, ${ }^{6}$ Department of Development and Regeneration, KU Leuven, Leuven, Belgium
\end{abstract}

Gait deficits in cerebral palsy (CP) are often treated with a single-event multi-level surgery (SEMLS). Selecting the treatment options (combination of bony and soft tissue corrections) for a specific patient is a complex endeavor and very often treatment outcome is not satisfying. A deterioration in $22.8 \%$ of the parameters describing gait performance has been reported and there is need for additional surgery in $11 \%$ of the patients. Computational simulations based on musculoskeletal models that allow clinicians to test the effects of different treatment options before surgery have the potential to drastically improve treatment outcome. However, to date, no such simulation and modeling method is available. Two important challenges are the development of methods to include patient-specific neuromechanical impairments into the models and to simulate the effect of different surgical procedures on post-operative gait performance. Therefore, we developed the SimCP framework that allows the evaluation of the effect of different simulated surgeries on gait performance of a specific patient and includes a graphical user interface (GUI) that enables performing virtual surgery on the models. We demonstrated the potential of our framework for two case studies. Models reflecting the patient-specific musculoskeletal geometry and muscle properties are generated based solely on data collected before the treatment. The patient's motor control is described based on muscle synergies derived from pre-operative EMG. The GUI is then used to modify the musculoskeletal properties according to the surgical plan. Since SEMLS does not affect motor control, the same motor control model is used to define gait performance pre- and post-operative. We use the capability gap (CG), i.e., the difference between the joint moments needed to perform healthy walking and the joint moments the personalized model can generate, to quantify gait performance. In both cases, the CG was smaller post- then pre-operative and this was in accordance with the measured change in gait kinematics after treatment.

Keywords: cerebral palsy, muscle synergies, single event multilevel surgery, orthopedic interventions, capability gap, subject specific model 


\section{INTRODUCTION}

Cerebral Palsy (CP) is the most common cause of motor deficiency in young children with a prevalence of 2-3 cases per 1,000 live births (Fairhurst, 2012; Colver et al., 2014; Graham et al., 2016). Due to lesions in the developing brain, children with CP display motor disabilities that vary greatly in presentation and severity. While $\mathrm{CP}$ is not a progressive disease, with time, secondary symptoms might arise, such as bony deformities and muscle contractures. Alongside increasing pain and fatigue, these symptoms can pose severe limitations to the quality of life and independence of the patients (Hanna et al., 2009; Opheim et al., 2009). Nowadays, several orthopedic treatments, often in combination with physical therapy and orthoses, are available and aim at improving the functionality and therefore quality of life of these patients (Fairhurst, 2012; Narayanan, 2012; Fitoussi and Bachy, 2015; Strobl et al., 2015; Nieuwenhuys et al., 2016).

For ambulatory patients, orthopedic treatments usually aim at improving walking speed and stability, at reducing the need of walking aids and at mitigating or preventing fatigue and pain (Narayanan, 2012). The selection of the most appropriate surgical treatment is a complex endeavor that nowadays is mainly based on the clinical assessment of the patient, integrated 3D gait analysis and medical imaging (Molenaers et al., 2001; Strobl et al., 2015). The outcome, however, is not always as desired and studies reported a deterioration in $22.8 \%$ of the parameters used to describe gait performance after surgery (Filho et al., 2008). In $11 \%$ of the cases, additional surgeries are needed to improve the functional outcome, although, this can be as high as $32 \%$ when no gait analysis is used to support the decision-making process (Wren et al., 2009).

It is therefore of the utmost importance to identify the parameters that determine the success of an orthopedic intervention (Hersh et al., 2002; Arnold et al., 2006a; Niiler et al., 2007; Fox et al., 2009; Reinbolt et al., 2009; Hicks et al., 2011; Schwartz et al., 2013, 2016; Mansouri et al., 2016; Galarraga et al., 2017). This would allow making pre-operative predictions in order to guide the decision-making process toward the most effective treatments in terms of functional outcome. Several studies applying statistical approaches and more recently machine learning methods to explore these relationships (Hersh et al., 2002; Reinbolt et al., 2009; Hicks et al., 2011; Schwartz et al., 2013, 2016) have been quite successful in predicting the improvement or non-improvement of a few selected outcome indicators when dealing with selected surgeries. However, existing methods do not produce a comprehensive outcome prediction and do not account for combinations of different surgeries. Notably, Galarraga et al. (2017) developed a method based on dimension reduction and multiple linear regression to predict lower limb kinematics for a large number of surgical procedures. All these methods, however, have the drawback that they are black box methods and therefore do not allow investigating the mechanisms relating outcomes in motor function to the specific interventions (Halilaj et al., 2018). On the other hand, methods relying on musculoskeletal models and computational simulations are often suggested to have the potential to identify the causal relation between individual impairments, their interactions and the treatment outcome (Morrison et al., 2018).

To introduce simulation-based decision-supporting tools into clinical practice, a few obstacles have yet to be overcome. One of the major obstacles in this respect is the need for a representative translation of the neuromusculoskeletal dysfunctions of the patients (i.e., the altered musculoskeletal geometry, musculoskeletal parameters, and altered neural control) into the musculoskeletal models. The need to account for the musculoskeletal deformities of the individual $\mathrm{CP}$ patient and the bony deformities in particular, dictates the use of subject-specific musculoskeletal models when generating dynamic simulations of $\mathrm{CP}$ gait. In this respect, the added value of magnetic resonance imaging (MRI) based models has been extensively demonstrated (Scheys et al., 2011a,b; Bosmans et al., 2016).

The altered muscle parameters (i.e., muscle contracture and weakness) in patients with $\mathrm{CP}$ compared to a healthy population (Theis et al., 2016; Kruse et al., 2017; Kalkman et al., 2018) invalidates the use of scaled generic parameters. Appropriate parameter tuning capturing the patient-specific muscle properties is therefore needed. Several methods have been proposed for tuning and scaling musculoskeletal parameters (Van Campen et al., 2014; Modenese et al., 2016; Falisse et al., 2017). Nevertheless, most of these methods require an extensive amount of data collected based on a specific method (e.g., instrumented dynamometry) that is typically not available in the common clinical practice and might be difficult to apply in the case of neuromotor deficits.

The altered motor control of patients with $\mathrm{CP}$ is reflected in the use of aberrant coordination patterns of the muscles during gait compared to a healthy population (Steele et al., 2015). The concept of muscle synergies is an elegant way to summarize these coordination patterns and by comparing them between CP and typically developing (TD) children, altered motor control aspects have already been identified in terms of number of independent components and stride-by-stride variability (Steele et al., 2015; Kim et al., 2018). In addition, muscle synergies have already been used in the control of musculoskeletal models during dynamic simulations (Allen and Neptune, 2012; Sartori et al., 2013; Meyer et al., 2016). In healthy subjects, the muscle activations generating the observed muscle synergies are very similar to those generated when muscles are recruited independently according to an optimality criterion (De Groote et al., 2014). However, patients with CP exhibit different sets of muscle synergies with respect to a healthy population (Steele et al., 2015), thus highlighting the importance of including a subject-specific motor control model into the framework (Meyer et al., 2016; Sartori et al., 2017).

Literature results (Patikas et al., 2007) and a pilot study from our research group (Pitto et al., 2018), suggest that the same motor control model can be used to describe both the pre- and post-operative patient's condition. Therefore, the preoperative synergies may also be used for the simulations of the post-operative condition, as their composition remains mostly unchanged after a specific orthopedic treatment. The advantage of this approach is that it relies entirely on pre-operative 
data, thus making it suitable for the pre-operative decisionmaking process.

Apart from describing the patient-specific features in the modeling framework, also the specific therapeutic interventions (and multi-level surgeries in particular) need to be accounted for into the musculoskeletal model. Whereas the effect of muscle-tendon lengthening and muscle transfer on the momentgenerating capacity, lengths and velocities of the muscles have been described (Delp and Zajac, 1992; Arnold et al., 2006b), only few studies attempted a forward simulation aiming to predict the post-intervention outcome. For instance, two studies using musculoskeletal modeling and forward dynamics simulations (Fox et al., 2009; Mansouri et al., 2016) investigated the effect of a rectus femoris transfer surgery on the recovery of balance after a perturbation and on knee flexion in stiff knee gait in children with CP. While these studies represent a step forward in this direction, their scope remains quite restricted, accounting for only one kind of intervention and analyzing the effect on a single parameter.

Clinical use of simulation-based decision-supporting tools requires the definition of comprehensive parameters that relate to the functional improvement of the patient and therefore can be used as outcome measures to evaluate the effect of different interventions. In the field of assistive exoskeletons (Afschrift et al., 2014), the concept of the capability gap (CG) was introduced to represent the amount of support the exoskeleton had to provide in order to allow the patient to perform a given task. This concept can be translated to the estimation of the motor performance of the patient, before and after the simulated interventions, as a measure of "difficulty" in performing a desired motion, i.e., gait pattern of a TD child. By integrating the patientspecific impaired motor control, abnormal muscle properties, and/or altered musculoskeletal geometry, the changes in the CG after a simulated orthopedic treatment inform the clinician on how a specific intervention improves the ability of the child to adopt a TD gait pattern.

Within the SimCP project, a comprehensive simulationbased framework was developed to evaluate the functional effect of a therapeutic/surgical intervention in a specific patient with $\mathrm{CP}$, thereby assisting the most appropriate treatment selection (Figure 1). This framework relies on the creation of a personalized neuro-musculoskeletal model of the patient. In this model, the musculoskeletal geometry is obtained from imaging data. The framework then provides the tools to personalize the muscle parameters according to information collected during gait analysis and clinical examination. Furthermore, the motor control is personalized using EMG data collected during the treatment-planning phase. Thereafter, a Graphical User Interface (GUI) allows clinicians to simulate combinations of different multi-level surgical procedures. Finally, the functional performance (i.e., walking ability) of the patient can be quantified for different simulated post-operative conditions by evaluating the change in the predicted capability gap with respect to the preoperative condition. These operations rely only on experimental data collected pre-operative. In this manner, it is feasible to compare the effectiveness of a set of candidate treatments in improving the gait performance of the patient, thus supporting the clinical decision-making process and optimizing individual treatment outcome.

Throughout this article, the different building blocks composing the framework are described and two representative cases studies are introduced to assess the methods and elucidate the several steps.

\section{METHODS}

The goal of the SimCP framework is to predict gait performance following different candidate orthopedic treatments solely based on data collected before the treatment and the surgical plan. Pre-operative data includes gait analysis, clinical examination (e.g., documenting joint range of motion) and medical images. To this aim, the framework contains several building blocks (Figure 1). First, a personalized musculoskeletal model capturing the patient's musculoskeletal geometry (section Musculoskeletal Geometry) and muscle properties (section Muscle Parameters) is generated. Then, a description of the patient's motor control is added to this model (section Motor Control). Next, the orthopedic surgeries are simulated (section Surgery Simulation). Finally, the simulated post-operative gait performance is computed (section Capability Gap and Muscle Report). The framework also offers the possibility to investigate the contributions of the different impairments to gait performance (section Alternative Analyses). We illustrate the salient features of the SimCP framework using data from two representative patients (section Case Studies).

\section{Data Collection}

In order to provide the information needed for the complete personalization of the models, inputs from several sources are required. First, imaging data, such as CT scans and MRI-images, allow defining the musculoskeletal geometry. Second, three-dimensional gait analysis data, including marker trajectories and ground reaction forces as well as EMG signals from the most important muscles in the lower limb, are needed for the personalization of the muscle parameters and definition of the motor control model. Third, clinical examination reporting the passive range of motion of the patient contains useful information to refine the personalization of the muscle parameters.

\section{Musculoskeletal Geometry}

The musculoskeletal models are based on a generic SIMM (Motion Analysis Corp., Santa Rosa, CA) model and are composed of 14 bodies and 21 degrees of freedom that are actuated by 86 muscles. The musculoskeletal geometry of this generic model is adapted to reflect the patient's musculoskeletal geometry. The model is then further personalized by tuning the muscle-tendon parameters (section Muscle Parameters) and by adding a model of the patient's motor control (section Motor Control).

Musculoskeletal geometry is derived from MRI-images. The workflow to create the MRI-based models has been published previously (Scheys et al., 2008, 2011b). In short, bones of the lower limbs and pelvis are segmented using Mimics (Materialize, 


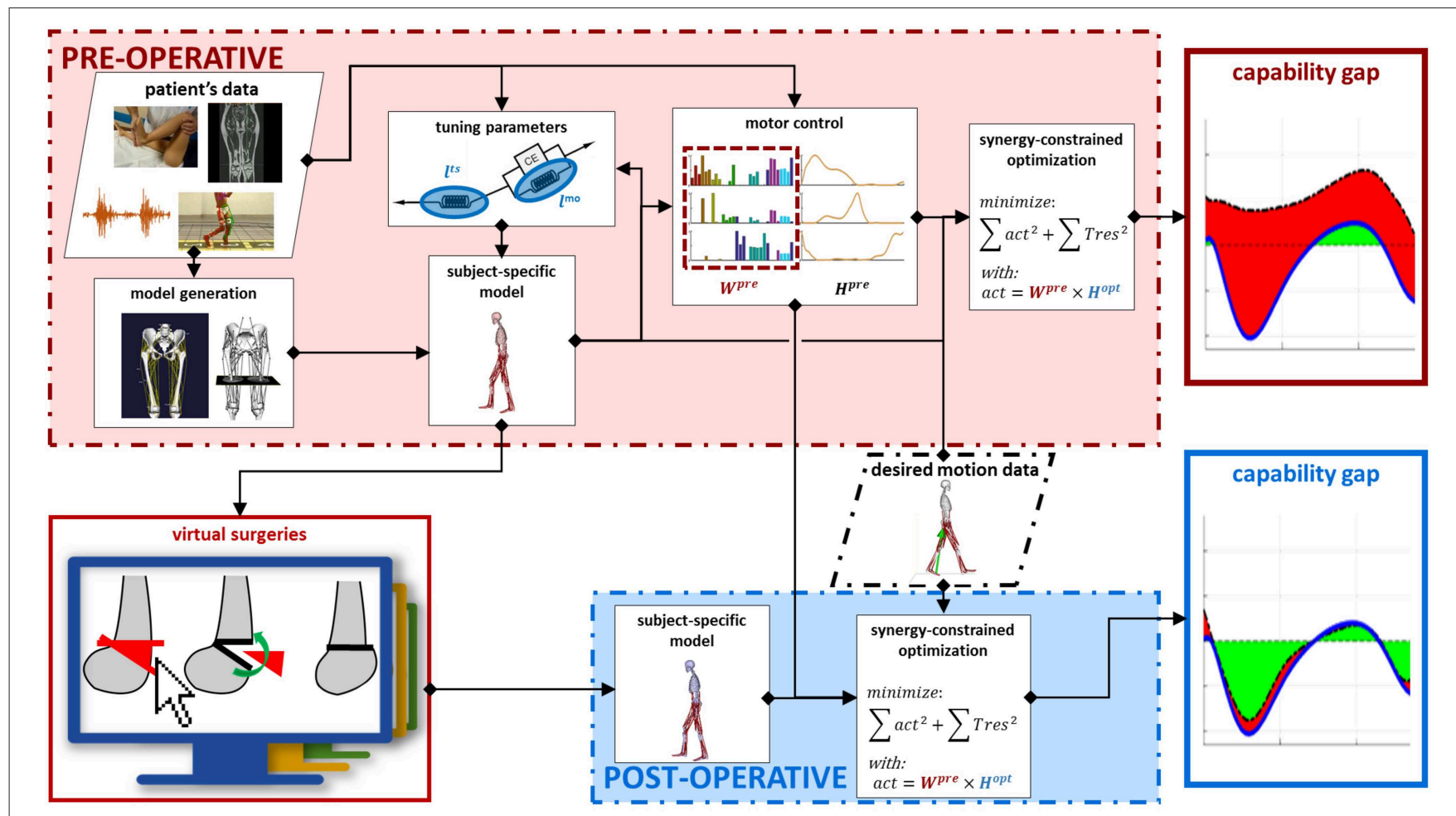

FIGURE 1 | Framework description. Data collected on the patient in the pre-operative phase are used as input to generate a pre-operative model with personalized musculoskeletal geometry and muscle parameters. Next, the motor control of the patient is modeled, using synergy decomposition analysis, based on pre-operative EMG and gait analysis data. Performing virtual surgeries on the pre-operative model generates a post-operative model. This takes into account the changes in the musculoskeletal parameters induced by the performed surgery. The motor control, on the other hand, is not affected by the surgery and the same motor control model computed in the pre-operative condition is used also for the post-operative condition. The gait performance of the patient can be computed in both the preand simulated post-operative conditions. In both cases, a synergy constrained optimization tries to match the joint moments relative to a desired motion while taking into account the constraints imposed by the model and by the motor control. The differences between the moments generated by the model and the desired moments are the capability gap, defining the gait performance.

Leuven, Belgium). Afterwards, anatomical reference frames, joint axes and muscle origin and insertion points are defined and a patient-specific musculoskeletal OpenSim model is created using MuscleSegmenter (Leuven, Belgium) and customized Matlab (The Mathworks, Natick, MA) scripts. The models with personalized musculoskeletal geometry are then imported into the SimCP framework.

Performing virtual surgery alters the musculoskeletal geometry and hence generates a new model, which is linked to the pre-operative model and appears in the post-operative model list. In this manner, each pre-operative model can be linked to multiple post-operative models, allowing for the exploration of different treatment options (see also section Surgery Simulation).

\section{Muscle Parameters}

Using preoperative data, we tune the two parameters of the Hill-type muscle model that have the largest influence on the simulated muscle force: muscle optimal fiber length and tendon slack length $\left(l^{m o}\right.$ and $\left.l^{t s}\right)$ (De Groote et al., 2010). Maximum isometric muscle forces are scaled based on the patient's body weight (Van Der Krogt et al., 2016; Kainz et al., 2018). Pennation angles are taken from the gait2392 model in OpenSim as they have a limited effect on simulated forces (Zajac, 1989).

Parameters are tuned using only the information from the pre-operative walking trials (marker trajectories, ground reaction forces and EMG) and, optionally, the clinical examination of the passive joint range of motion (Table 1). The underlying assumption of the procedure is that during gait the muscles generate the inverse dynamic moments with activations that are consistent with the measured EMGs and operate around their optimal fiber length. In other words, given the joint excursions during gait, muscles are not extremely short, as this would limit their force production given the muscle's force length relationship, nor too stretched, as this would induce excessive passive forces. In addition, it is assumed that at least part of the resistance encountered during the clinical examination at the extremes of the range of motion, is attributable to muscle passive force, i.e., muscles being at a length well above their optimal length.

To perform this tuning, we extended a static optimization problem since this allows us to optimize the fit between the computed activations and joint moments, and, respectively, the EMGs and inverse dynamic joint moments. Static optimization 
TABLE 1 | Subjects demographics and data from clinical examination.

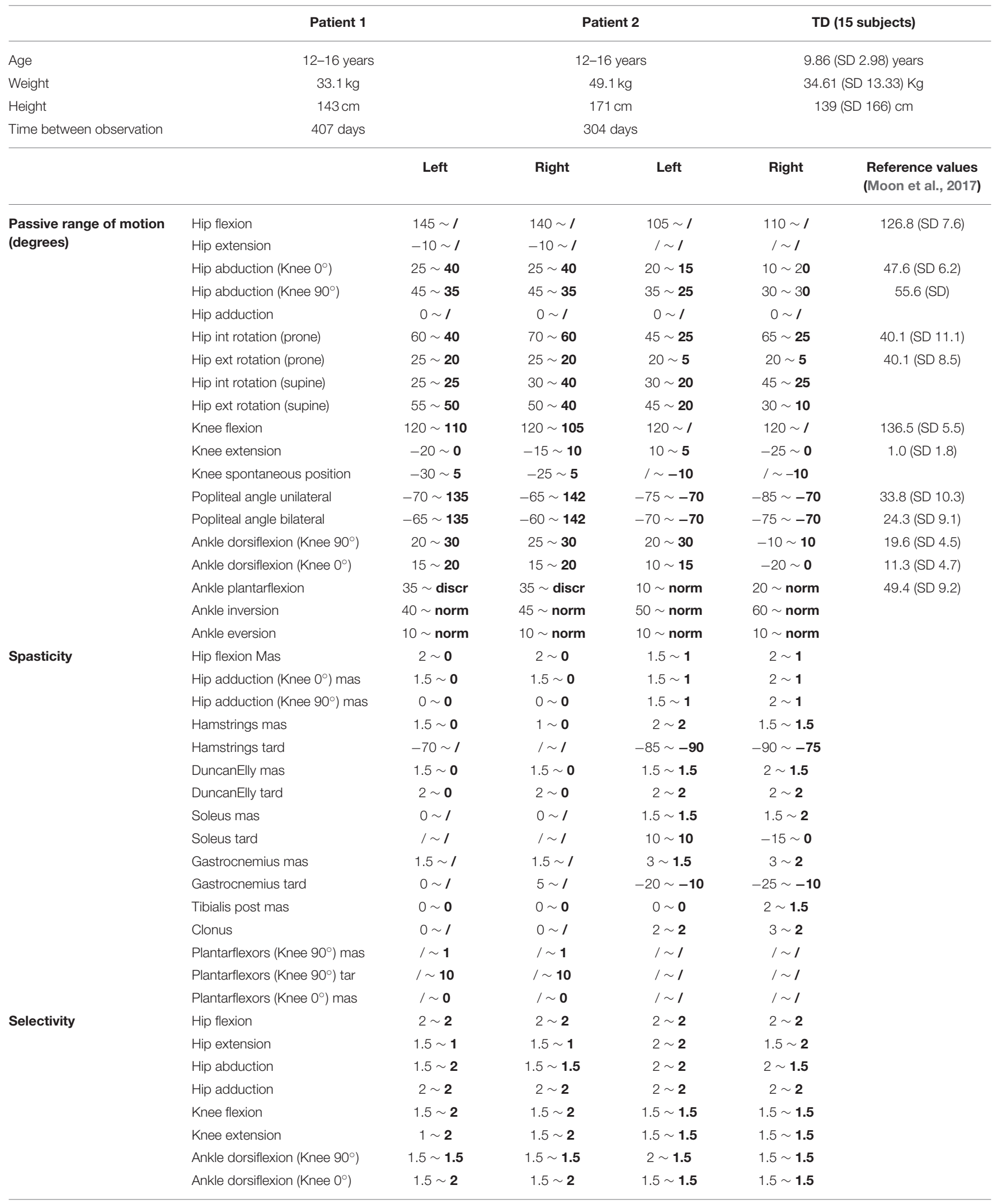




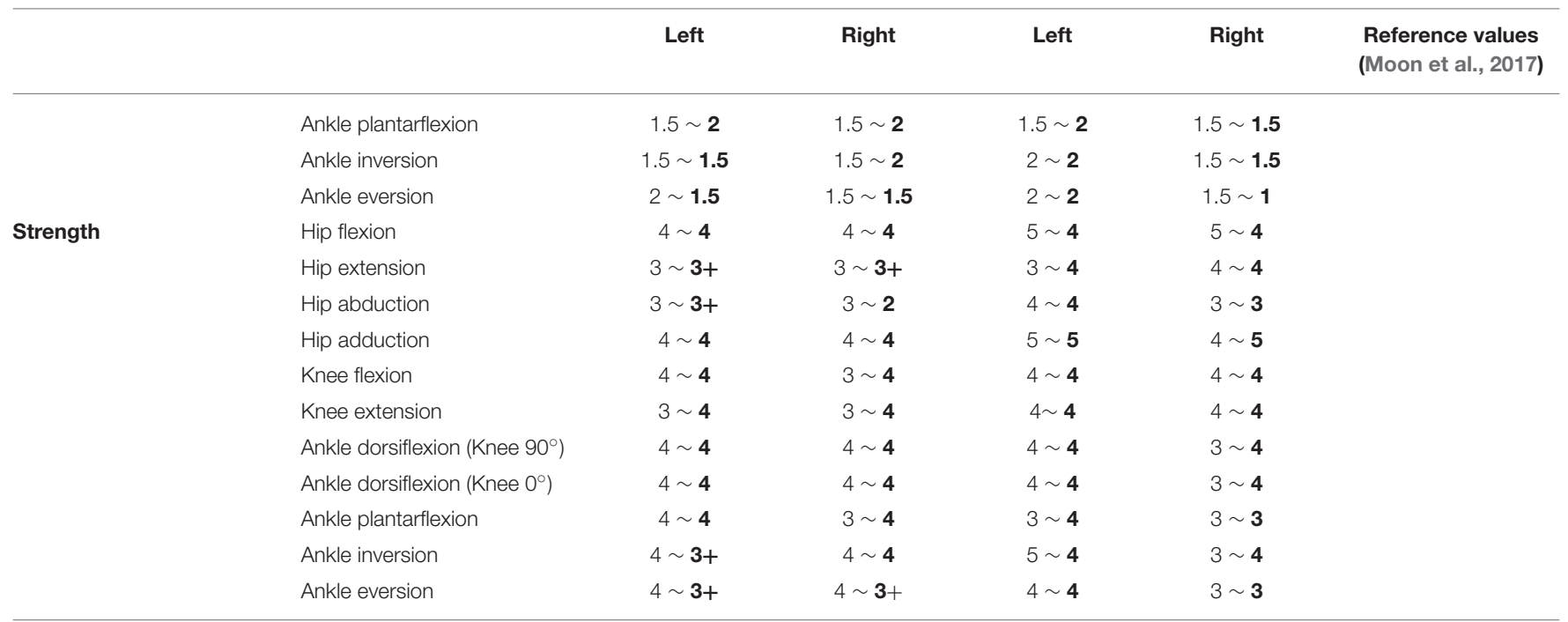

Information about the subjects included in the study. Data are collected from the pre- and post-operative clinical reports. For each entry, the first value is relative to the pre-operative condition, the second, in bold, is relative to the post-operative condition. "Mas" stands for manual Ashworth test (a score of 0 indicates no spasticity, a score of 4 maximal spasticity), "Tard" for Tardieu test (measured in degrees, defines the position where the spasticity limits the movement). In "Selectivity" a score of 2 indicates the maximum motor control selectivity.

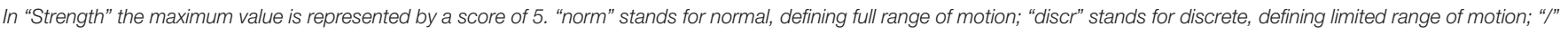
replaces values not present in the report. Reference values for TD subjects are obtained from Moon et al. (2017).

computes muscle activations that produce the inverse dynamic joint moments underlying a measured movement while optimizing a performance criterion (e.g., minimizing sum of activations squared). Here, we allow optimal fiber lengths and tendon slack lengths to change during the optimization while imposing constraints on the allowable muscle lengths that represent the tuning criteria described above. In contrast to the typical static optimization approach that is solved for each time frame separately, here all time frames are coupled to obtain a single set of muscle-tendon parameters. It is important to note that static optimization neglects muscle dynamics by assuming that tendons are rigid but allows accounting for the muscle force-length-velocity relationship (De Groote et al., 2016). A static optimization approach was preferred over a dynamic approach that accounts for muscle dynamics (De Groote et al., 2016) to limit computation times. The problem was then solved using the fmincon function in Matlab.

To cope with the scarcity of input data (i.e., data from a limited number of movements and a limited number of EMG signals), we decided to tune the parameters only in a set of major muscles $(M)$ (Table 2). In addition, we used a different level of detail when describing the force generated by these major and other muscles. For the major muscles, the forcelength relationship, derived from (De Groote et al., 2016), was taken into account (but not the force-velocity relationship). Hence, the generated force has an active component $\left(f^{L}\right)$, depending on normalized muscle fiber length $(\tilde{l})$ and muscle activation $(a)$, and a passive component, $\left(f^{P}\right)$ depending only on fiber length:

$$
{ }^{m} F_{i}={ }^{m} f_{\circ}\left[{ }^{m} a_{i}{ }^{m} f_{i}^{L}\left({ }^{m} \tilde{l}_{i}\right)+{ }^{m} f_{i}^{P}\left({ }^{m} \tilde{l}_{i}\right)\right], \forall m \in M,
$$

Where $f$ is the maximum isometric force the muscle can exert and the subscript $i$ defines the instant in time. For the remaining muscles $(N)$, the generated force is proportional to activation:

$$
{ }^{m} f_{i}={ }^{m} f_{0}{ }^{m} a_{i}, \quad \forall m \in N .
$$

Hence, the resulting estimation problem has the following structure. Optimization variables consist of muscle activations and reserve moments (for each joint $j$ ) during the gait cycle $\left({ }^{m} a_{i},{ }^{j} \tau_{i}^{R}\right)$, as well as the muscle parameters $\left({ }^{m} l^{m o},{ }^{m} l^{t s}\right)$. Reserve moments are generated by ideal actuators and are added to the muscle moments to guarantee that the inverse dynamic joint moments can be matched even when the muscles are not sufficiently strong. Since they are not physiological, their use is heavily penalized in the cost function to keep their contribution to a minimum:

$$
C^{S O}=\sum_{i}\left(\sum_{m} w_{1}\left({ }^{m} a_{i}\right)^{2}+\sum_{j} w_{2}\left({ }^{j} \tau_{i}^{R}\right)^{2}\right),
$$

Where $w_{1-2}$ are weighting coefficients that produced a proportional balance between muscle and residual activations (Hicks et al., 2015) in simple static optimization problems.

An additional penalty term is included in the cost function to ensure that the computed activations of the subset of muscles $(\varepsilon)$ for which EMG was collected (see Table 2) reflect the pattern of the measured data:

$$
C^{\epsilon}=\sum_{i} \sum_{m \in \epsilon} w_{3}\left({ }^{m} \sigma^{m} a_{i}-{ }^{m} \varepsilon_{i}\right)^{2}
$$

Where $\varepsilon$ represents the experimental EMG envelope. The scaling factor $\sigma$ was introduced as an optimization variable to impose 
TABLE 2 | List of muscle subsets.

\begin{tabular}{|c|c|c|c|c|}
\hline \multirow{2}{*}{$\begin{array}{l}\text { Tuned muscles } \\
\text { Glut Max } 1\end{array}$} & \multicolumn{2}{|c|}{ EMG muscles } & \multicolumn{2}{|r|}{ Passive range of motion } \\
\hline & EMG channel & Muscle name & Measurement & Muscles measured \\
\hline Glut Max 2 & Rectus Fems & Rectus Fem & & Hip \\
\hline Glut Max 3 & Vast Lat & Vast La & Flexion & Glut Max1, Glut Max2, Glut Max3, \\
\hline Glut Med 1 & Bic Fem & Bic Fem Ih & & Glut Med1, Glut Med2, Glut Med3 \\
\hline Glut Med 2 & Hamstring Med & Semimembr, Semitend & Extension & Iliacus, Psoas \\
\hline Glut Med 3 & Tibialis anterior & Tibialis Ant & Abduction $0^{\circ}$ & Add Mag 2, Add Mag 3, Add Long \\
\hline Add Long & Gastrocnemius & Gastroc Med, Gastroc Lat & Int Rot Sup & Glut Med1, Glut Med2, Glut Med3 \\
\hline Add Mag 2 & Soleus & Soleus & Int Rot Pro & \\
\hline Add Mag 3 & Gluteus & Glut Med 2 & Ext Rot Sup & \\
\hline Tensor FL & & & Ext Rot Pro & \\
\hline Gracilis & & & & Knee \\
\hline Semimembr & & & Flexion & Rectus Fem, Vast Int, Vast Med, Vast Lat \\
\hline Semitend & & & Rectus Fem & \\
\hline Bic Fem Ih & & & Extension & Semimembr, Semitend, Gracilis, Bic Fem Ih, Bic Fem sh \\
\hline Bic Fem sh & & & Popl Ang Uni & \\
\hline Sartorius & & & Popl Ang Bi & \\
\hline Rectus Fem & & & & Ankle \\
\hline Vast Med & & & Dorsiflex $\mathrm{Kn} 0^{\circ}$ & Soleus, Gastroc Lat, Gastroc Med \\
\hline Vast Int & & & Dorsiflex Kn $90^{\circ}$ & \\
\hline \multicolumn{5}{|l|}{ Vast Lat } \\
\hline \multicolumn{5}{|l|}{ Gastroc Med } \\
\hline \multicolumn{5}{|l|}{ Gastroc Lat } \\
\hline \multicolumn{5}{|l|}{ Soleus } \\
\hline \multicolumn{5}{|l|}{ Iliacus } \\
\hline Psoas & & & & \\
\hline
\end{tabular}

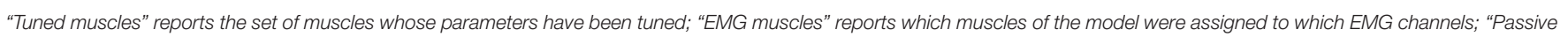
Range of motion" reports the clinical examination measures included into the framework and which muscles were measured in each position.

similarity between activations and EMG patterns irrespective of signal amplitude since, in the absence of maximum voluntary contraction tests, the relation between signal amplitude and muscle activation cannot be accurately derived.

The cost function was minimized subject to the following constraints. A first set of constraints describes that the muscles should produce the inverse dynamic joint moments:

$$
\sum_{m \in M}{ }^{j m} r_{i}{ }^{m} F_{i}+\sum_{m \in N}{ }^{j m} r_{i}{ }^{m} f_{i}+{ }^{j} \tau_{i}^{R}-{ }^{j} \tau_{i}^{I D}=0,
$$

Where ${ }^{j} \tau_{i}^{I D}$ are the desired joint moments from inverse dynamics and ${ }^{j m} r_{i}$ is the moment arm of muscle $m$ with respect to joint $j$ at time $i$.

A second set of constraints imposes bounds on the muscle fiber lengths during gait and the clinical exam of the range of motion. To ensure that normalized muscle fiber lengths during gait are within 0.4 and 1.5 , we constrain the minimal and maximal fiber lengths. In addition, to ensure that muscles operate around their optimal length during gait, we constrain the maximal fiber length to be above and the minimal fiber length to be below optimal fiber length:

$$
1<\max _{i}\left({ }^{m} \tilde{l}_{i}\right) \leq 1.5
$$

$$
0.4 \leq \min _{i}\left(\tilde{l}_{i}\right)<1, \quad \forall m \in M
$$

Maximal normalized fiber lengths during the clinical exam $\left(\tilde{l}^{R}\right)$ should be in the range where passive force is generated:

$$
1<m \tilde{l}^{R} \leq 1.5, \quad \forall m \in R
$$

Where $R$ defines the subset of muscles for which the length is computed using information from the clinical passive range of motion examination (Table 2).

To impose that muscles were stretched to a level where they generated considerable passive force during the clinical exam, we added a penalty term to the cost function:

$$
C^{R}=\sum_{m \in R} w_{4}\left(m \tilde{l}^{R}-1.5\right)^{2},
$$

Within our formulation, the passive force exerted by a muscle stretched at $1.5 l^{m o}$ is around $0.5 F$. While a significant variation in passive force is present between muscles (Prado et al., 2005) and further variations are induced by CP (Kalkman et al., 2018), we made this simplifying assumption to allow for the selection of different sets of muscles without increasing the complexity of the tuning procedure. 
Combining, (Equations 3, 4, and 9) and, the final cost function becomes

$$
C^{S O}+C^{\epsilon}+C^{R}
$$

Input data ${ }^{j} \tau_{i}^{I D},{ }^{j m} r_{i}$, and muscle-tendon lengths ${ }^{m} l_{i}^{m t}$ are computed based on the personalized musculoskeletal models using OpenSim's analysis tools, specifically Inverse Kinematics, Inverse Dynamics and Muscle Analysis using the gait data as input. Therefore, muscle moment arms are defined using the generalized force method (Sherman et al., 2014). Muscle fiber lengths $(l)$ and normalized fiber length $(\tilde{l})$ during gait were computed according to a Hill-type model assuming a rigid tendon:

$$
\begin{aligned}
& { }^{m} l_{i}=\left({ }^{m} l_{i}^{m t}-{ }^{m} l^{t s}\right) / \cos (\alpha), \\
& { }^{m} \tilde{l}_{i}={ }^{m} l_{i} /{ }^{m} l^{m o}, \quad \forall m \in M,
\end{aligned}
$$

Where $\alpha$ is the pennation angle. Joint positions during the clinical exam to test the passive range of motion are derived from the description of the test and are used to compute the maximum musculotendon lengths, muscle fiber lengths and normalized fiber lengths reached during the test for the subset of muscles $R$ $\left(l^{m t R}, l^{R}\right.$ and $\tilde{l}^{R}$, respectively).

The estimated parameters $l^{m o}$ and $l^{t s}$ are incorporated into the subject's model and will define the force-length relationships of the muscles included in the set $M$ during the following analyses.

\section{Motor Control}

In cerebral palsy, the ability to selectively recruit muscles is reduced. Therefore, we describe impaired motor control by imposing the pathological muscle activation patterns when computing gait performance. Muscle activation patterns are derived from pre-operative EMG data collected during walking using synergy analysis. For the motor control model to be useful in our simulations, it has to account for the activations of all the muscles. However, EMG data are collected from only a small subset of muscles (typically $<10$ ) while the musculoskeletal model contains many more muscles (typically more than 40 ). A multi-step procedure is proposed to derive muscle coordination for all muscles in the model based on EMG data from a limited number of muscles (cfr. Meyer et al., 2016).

First, muscle synergies are derived from the pre-operative EMG data acquired during three gait cycles to define the complexity of the patient's motor control by the number of synergies $\left(N^{s}\right)$ using non-negative matrix factorization (NNMF) (Lee and Seung, 1999). The input matrix of EMG signals has dimensions $N^{\epsilon} \times N^{i}$ where $N^{\epsilon}$ is the number of muscles and $N^{i}$ is the number of time instants. The output of the synergy analysis consists of two matrices: a $N^{s} \times N^{i}$ matrix $H$ containing the activation timing profiles of each synergy and a $N^{\epsilon} \times N^{s}$ matrix $W$ containing the weight vectors specifying how much an individual muscle is activated by each synergy. The matrices $W$ and $H$ are computed such that the product $W H$ best approximates the original input matrix for a predefined number of synergies $N^{s}$. We quantified $N^{s}$ using a bootstrapping procedure such that the percentage of the original signal explained by the synergies is above a predefined threshold (Cheung et al., 2009). Both the EMG signal and the $H$ matrix were consistently resampled 500 times with replacements, using Matlab function datasample. The resampled matrices have the same dimension of the original ones and the same time instants of the original matrices can appear more than once in the resampled one. The variability accounted for (VAF) of these resampled signals by the synergies extracted from the original signal is computed. $N^{s}$ is the lowest number of synergies for which VAF is higher than $90 \%$ for at least $95 \%$ of the resampled signals. The number of synergies $N^{s}$ is used in the subsequent steps.

Next, an EMG-informed static optimization analysis is performed on the patient's pre-operative gait data, where the optimization variables are ${ }^{m} a_{i},{ }^{j} \tau_{i}^{R}$, and ${ }^{m} \sigma$. The cost function is obtained by combining (Equations 3 and 4):

$$
C^{S O}+C^{\epsilon}
$$

The $C^{\epsilon}$ term enables us to account for the pathological characteristics of muscle activations, such as antagonistic muscle co-contractions, which are very common in children with $\mathrm{CP}$. This cost is minimized subject to constraints describing the equilibrium between inverse dynamic and muscle moments (Equation 5).

Finally, muscle synergies are extracted by performing a new NNMF on the muscle activations computed with static optimization. The number of synergies for this analysis is $N^{s}+1$. The extra synergy is included to take into account muscles for which no surface EMG was collected. For instance, Allen and Neptune (2012) found that a synergy including predominant contributions from the iliacus and psoas muscles is needed to control a 3D model during walking, and EMG from these muscles is typically not acquired. The result is a set of muscle synergies ( $W^{p r e}$ and $H^{p r e}$ ) that define the motor control model of the patient and describe the activations of all the muscles. This motor control model is later used in the computation of the gait performance of the patient (section Capability Gap and Muscle Report).

\section{Surgery Simulation}

We developed a set of virtual surgeries and a GUI that allow to directly manipulate the musculoskeletal models by leveraging the Matlab-OpenSim application programming interfaces. The surgeries implemented in the current version of the GUI are Extension and Derotation Osteotomy, Derotation Osteotomy, Muscle Transfer, Patella Advancement and Botulinum Toxin Injection. Only the Muscle Transfer and Botulinum Toxin Injections influence the muscle parameters.

In an Extension and Derotation Osteotomy (Figure 2B), two cutting planes define the bone wedge for removal (Lenhart et al., 2017). In the GUI, the pose of the cutting planes on the desired bone can be defined. The wedge is removed and the remaining bone segments are then reconnected by joining the cutting planes. The intra-segment rotation perpendicular to the cutting planes and the translation along the cutting plane can be specified by the user. Based on bony landmarks, important morphometric information, such as anteversion angle and neck 


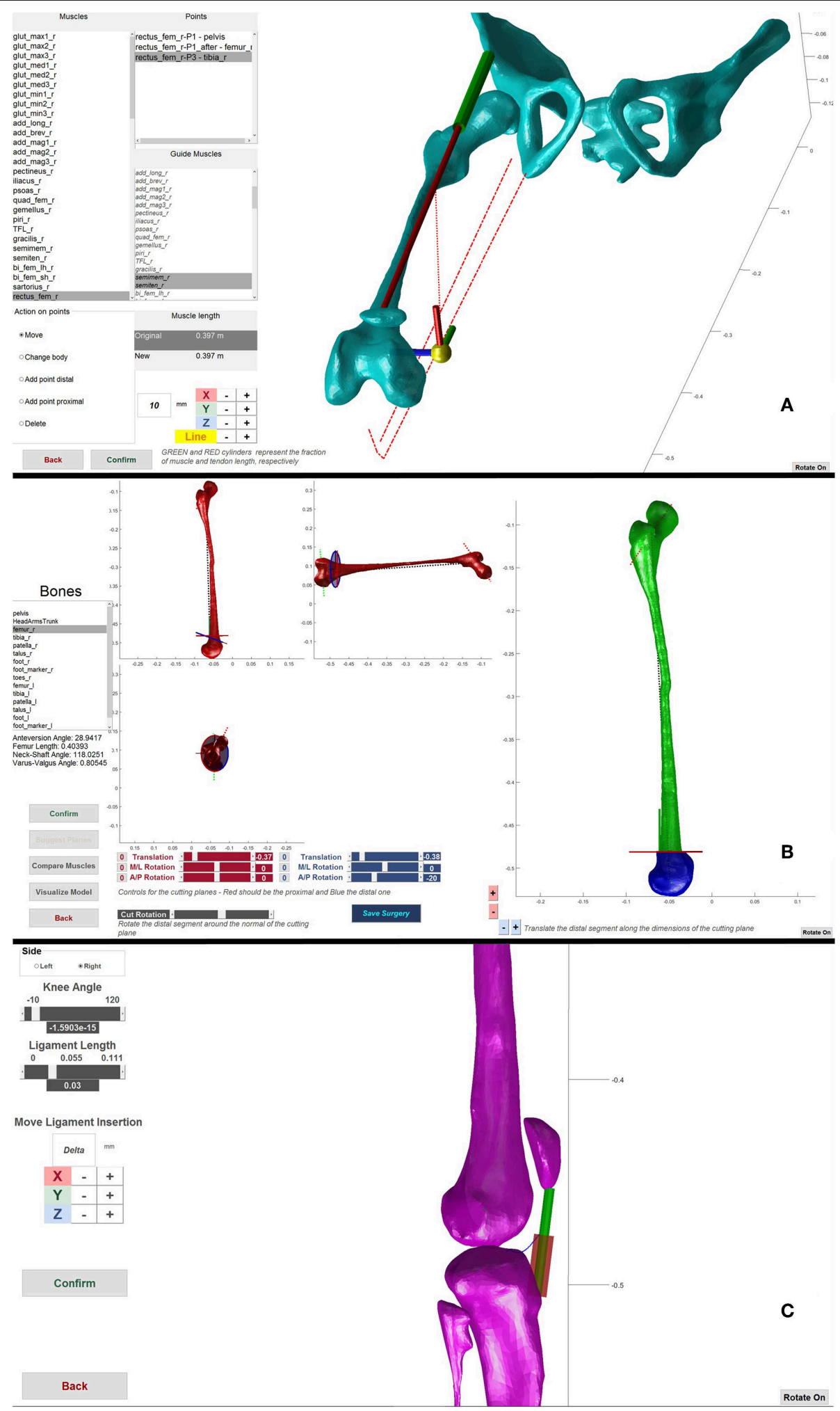

FIGURE 2 | Framework components. (A) Muscle transfer surgery. Used to change the path of a muscle. The lines of action of other muscles are visualized as guide to help during the transfer. (B) Extension and Derotation osteotomy surgery. Two sets of scrollbars define the pose of the two cutting planes (red and blue in the left side figures) that define the wedge of bone to be removed. After wedge removal, the two segments are brought in contact and it is possible to rotate and translate the distal part to correct for abnormal anteversion angles. (C) Patella advancement surgery. It is possible to define the new length of the patella ligament and/or move its insertion on the tibia, during the operation the ligament in the new configuration is shown as a red cylinder. 
shaft angle of the simulated post-operative bone configuration are visualized in real-time to guide the user in performing the virtual surgery. The Derotation Osteotomy surgery is implemented in a manner similar to the Extension and Derotation Osteotomy but involves only a single cutting plane on the desired bone. After cutting the bone, the distal part of the bone can be rotated and translated with respect to the proximal part of the bone to correct for bony deformities. Within the Muscle Transfer tool (Figure 2A), we provide several options to modify the muscle geometry. These options include adding, removing, translating and changing the muscle attachment, insertion and via points. The resulting change in musculotendon length is translated into a change in tendon slack length. The underlying assumption is that muscle transfer surgeries do not directly affect the muscle fiber architecture, but change the length of the tendon either by removing part of the tendon, lengthening the tendon, or by transferring it to the tendon of another muscle. Patella Advancement (Figure 2C) can be performed by either changing the length of the patella ligament, or by transferring the attachment of the ligament on the tibia to a new position. Patella movement is defined as a function of knee flexion angle. After changing the ligament attachment or length, the new path of the patella is determined through an optimization procedure. This optimization procedure defines the rotation and the translations needed to represent the patella movement on the plane perpendicular to the knee joint axis. This optimization finds the patella movement that results in the most constant distance between two points on the patella, one proximal and one distal, and the femur surface throughout the motion while maintaining the patella ligament length constant. Botulinum Toxin Injections are modeled by a decrease in the injected muscle's maximum isometric force. This is a highly simplified representation of what botulinum toxin injections do to the muscle and further research is needed to refine this procedure in our model.

Any change applied to a given model results in a new postoperative model, which can be saved for future use.

\section{Capability Gap and Muscle Report}

As previously introduced, the main outcome of the present framework is the capability gap. The capability gap is the difference between the joint moments needed for performing a "desired" motion, i.e., TD walking, and the joint moments the personalized model of the patient (this can be either a pre- or post-operative model) can generate. We use a synergy-based static optimization approach to compute the capability gap. Here, the reserve moments appearing in the moment equilibrium function (Equation 5) represent the torque deficit and hence the capability gap. Subject-specific musculoskeletal geometry and muscle parameters are described in the musculoskeletal model. Impaired motor control is imposed through additional constraints on the activations based on the patient's muscle synergies.

The desired motion used for the computation is derived by scaling average TD walking data to the patient's dimensions (Table 1). First, TD kinematics are imposed to a generic model that was scaled to the patient and corresponding $3 \mathrm{D}$ marker trajectories are extracted. The magnitude of the ground reaction forces is scaled based on mass and their point of application, expressed in the foot reference frame, is scaled based on body height. Successively, using the marker trajectories and ground reaction forces, the joint moments required for the personalized model to perform the desired motion are computed by an inverse kinematics and inverse dynamics analysis. In addition, corresponding muscle moment arms and musculotendon lengths are computed. By tracking marker trajectories consistent with TD walking instead of imposing TD joint kinematics directly to the musculoskeletal model, we avoid that the presence of bony deformities leads to unrealistic gait patterns. As an example, if the femoral neck anteversion is $30^{\circ}$ higher than normal, imposing TD kinematics to the personalized model would result in a gait pattern with the knee and foot pointing outwards by about $30^{\circ}$, whereas if we track the marker trajectories the knee and foot will point forwards.

Afterwards, the synergy constrained static optimization is performed. The cost function to be minimized is $C^{S O}$ as defined by Equation (3). The moment equilibrium (Equation 5) has to be satisfied. Inputs to Equation $(5),{ }^{j} \tau_{i}^{I D},{ }^{j m} r_{i}$, and ${ }^{m} \tilde{l}_{i}$, are computed based on the patient model and a TD walking pattern as described above. Instead of solving for independent muscle activation patterns, we now solve for synergy activation patterns $H^{o p t}$. The optimization variables are hence $H_{i}^{o p t},{ }^{j} \tau_{i}^{R}$ and ${ }^{m} W$, which is a deviation from the pre-operative synergy weights (see below). Individual muscle activation patterns (including both subsets $N$ and $M$ ) are then computed from the synergy activation patterns using the synergy weight vectors that describe the patient's motor control $W^{\text {pre }}$ (see section Motor Control):

$$
{ }^{m} a_{i}=\left(W^{p r e}+\Delta^{m} W\right) \times H_{i}^{o p t},
$$

The same muscle co-contraction patterns ( $W^{\text {pre }}$ ) are used to compute the pre- and post-operative CG, since we hypothesize that the orthopedic intervention does not alter muscle cocontraction patterns. Hence, we assume that the neural system will respond to the altered musculoskeletal geometry by changing the timing and magnitude of the pre-operative activation patterns $H$. A small deviation $(W)$ from the original weights is allowed because the synergy matrices computed using NNMF typically do not capture $100 \%$ of the signal variability. In our formulation, $W$ is normalized so that the maximum value in each vector equals one and $W \leq 0.05$.

Because of the altered musculoskeletal geometry, muscle parameters and synergy-based constraints on muscle activations, it is likely that the muscles cannot generate the TD joint moments. The non-selective motor control imposed by the synergy weights might impose antagonistic co-contractions, hindering the moment generating capacity of a muscle. Alternatively, muscles could be excessively stretched when imposing TD gait kinematics and generate high passive forces. A considerable contribution from the residual actuators might thus be required to satisfy the moment equilibrium. The magnitude of the residuals moments required to match the desired moments defines the capability gap. 
The CG is represented graphically as a function of the gait cycle for the different degrees of freedom (Figure 3A) and is quantified for each degree of freedom:

$$
{ }^{j} C G=\sum_{i}\left|{ }^{j} \tau_{i}^{R}\right| / \sum_{i}\left|{ }^{j} \tau_{i}^{I D}\right|,
$$

Furthermore, we provide a muscle report (Figure 3B) summarizing the intervals during which muscles operate with excessively short or long fiber lengths. Excessively stretched muscles are those whose passive force is $>0.5$ times their maximum isometric force (corresponding to $\tilde{l}$ larger than $\left.1.5 l^{m o}\right)$. Muscles active at short lengths are those whose activations are $>0.25$ when operating at normalized fiber lengths smaller than 0.6, meaning that they are producing relatively little force.

\section{Alternative Analyses}

This framework is originally intended to work with the personalized neuro-musculoskeletal models as described above but it is also possible to exclude one or more of the personalization blocks. In fact, this procedure can be of value when assessing the importance of the different factors contributing to the impairment, as highlighted in section Case Studies. It is for instance possible to import a scaled, generic model, and perform the same analyses and surgeries, as envisaged for the personalized models, in order to evaluate the importance of the bony deformities in defining the impairment of the patient. Alternatively, it is possible to investigate alternative causes of the functional impairment by excluding the muscle force/length relationships or the constraints imposed by the motor control on the muscle activations when computing the gait performance (CG).

\section{Case Studies}

We analyzed two representative patients with the proposed framework (Table 1). Both were diagnosed with diplegic CP and underwent SEMLS. For both patients, MRI images were acquired prior to the intervention (for details on the protocol, see Bosmans et al., 2014). A standardized clinical examination protocol (Desloovere et al., 2006) was conducted to evaluate the level of spasticity, strength, selectivity and range of motion. Three-dimensional gait analysis was performed before and after the intervention. Each participant was equipped with a set of reflective markers using the Vicon Plug-in-Gait marker set for lower limbs. Using a 10-15 camera motion capture system (Vicon Motion Systems, Oxford, UK) and two force plates (AMTI, Watertown, MA, USA), marker trajectories and ground reaction forces were collected during one static trial and at least three walking trials at self-selected walking speed. EMG signals were collected (Zerowire, Cometa, Italy) from eight major muscles per leg (rectus femoris, vastus lateralis, biceps femoris long, medial hamstrings, tibialis anterior, gastrocnemius, soleus, and gluteus medius). The local ethical committee approved all procedures, and written informed consent was obtained from the parents of the children prior to participation.

We created pre-operative personalized models using the experimental data (MRI, 3D gait analysis and clinical examination report) and post-operative personalized model by performing virtual surgeries according to the surgical plan of the actual intervention. For Patient 1 the following interventions were modeled: bilateral rectus femoris transfer, distal femur extension, and derotation osteotomy, patella advancement. Patient 1 also received a derotation of the tibia, but this was not modeled due to the fact that the MRI from which the musculoskeletal model was built did not include images of the feet and distal tibiae. For Patient 2 the following interventions were modeled: bilateral rectus femoris transfer, left distal femur derotation, right distal femur extension, and derotation osteotomy, patella advancement, right gastrocnemius and psoas release.

To simulate the Extension and Derotation Osteotomy intervention, the angle between the two cutting planes in the femur was modeled based on the knee extension deficit observed when testing the passive range of motion. For the femur Derotation Osteotomy, the anteversion angle was corrected to be equal to $0^{\circ}$ in the simulated post-operative model in agreement with information provided by the orthopedic surgeons. Patella advancement was modeled by shortening the patella ligament by $2 \mathrm{~cm}$, as reported in the surgical plan. Rectus Femoris Transfer was modeled in two steps. First, a via point in the femur reference frame was introduced in the middle of the muscle-tendon unit. Second, the insertion site was transferred to the semitendinosus tendon, while keeping the original length of the musculotendon unit unchanged. Our approach replicates the surgical procedure in which the rectus femoris is detached and reattached distally but left attached proximally, thus maintaining its function as a hip flexor. Muscle Release interventions were modeled by completely removing the muscle contribution from the generated moment, i.e., by setting the maximum isometric muscle force to zero. Although both patients additionally received botulinumtoxin injections, these were not included in the postoperative model given that their effect can be considered small given the time between the pre- and post-operative observations (10 and 13.5 months).

We compared the predicted motor performances of the patients in terms of the capability gap for the pre-and postoperative conditions (Figure 3). We tested the effect of different treatment options on the capability gap of the right leg of Patient 2 (Figure 4). This was done by creating different models with different angles of the cutting planes defining the extension osteotomy $\left(20^{\circ}\right.$ and $\left.25^{\circ}\right)$ as well as two different shapes of the wedge of bone (triangular and trapezoidal). We also investigated the effect of including/excluding the altered muscle parameters and motor control on the predicted gait performance (Figure 5) in the pre-operative condition. When excluding altered motor control from the analysis, muscle activations could vary independently for all muscles. Finally, we tested the ability of our framework to predict post-operative performance by comparing the predicted gait performance (CG) with the gait performance quantified based on the 3D gait analysis performed before and after the intervention (Figure 6). We analyzed the root mean square errors between patient and TD kinematics, corresponding to the Gait Variable Scores and Gait Profile Scores (Baker et al., 2009). 


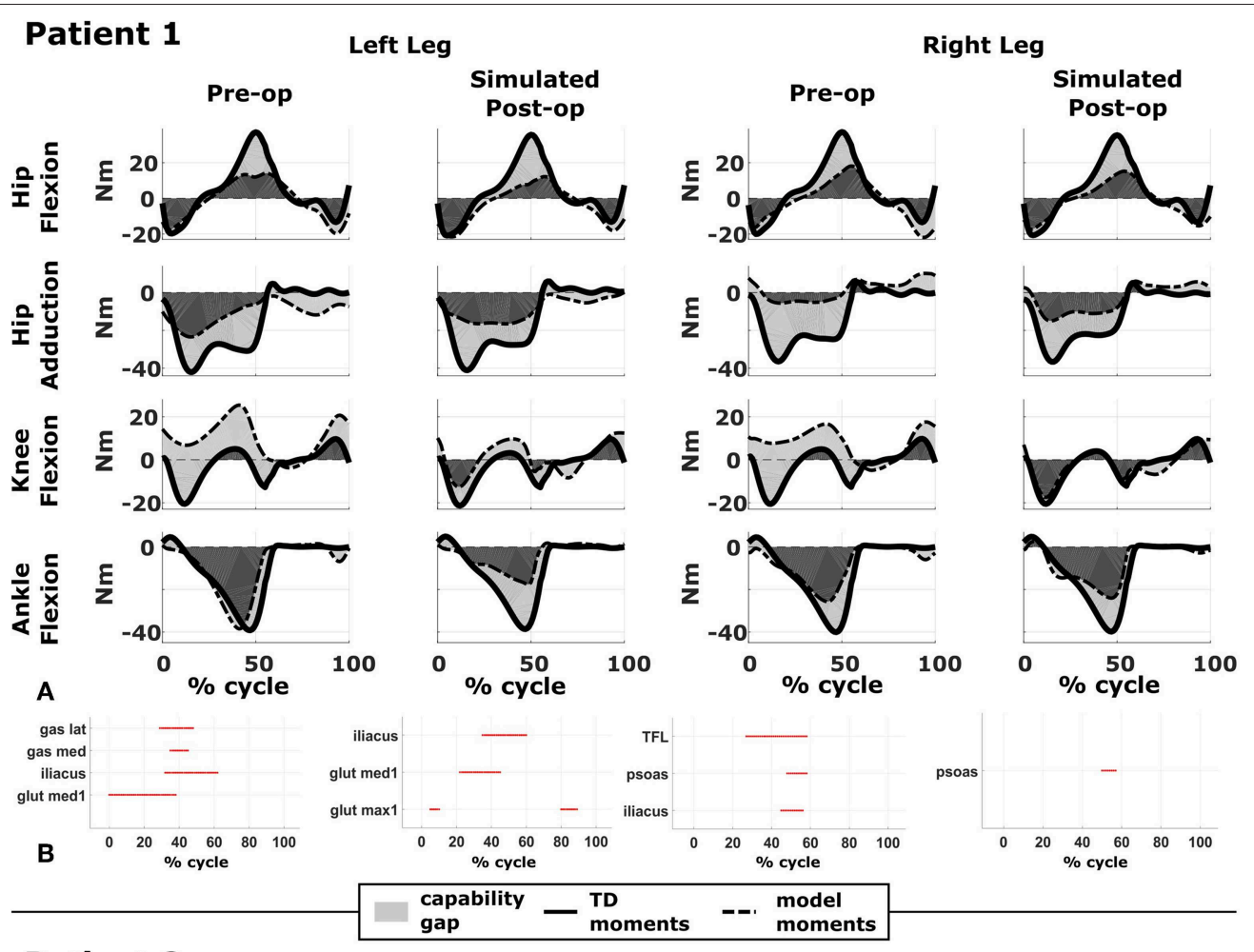

$\begin{array}{lll}\text { Patient } 2 \text { Left Leg Leg } & \text { Right Leg }\end{array}$

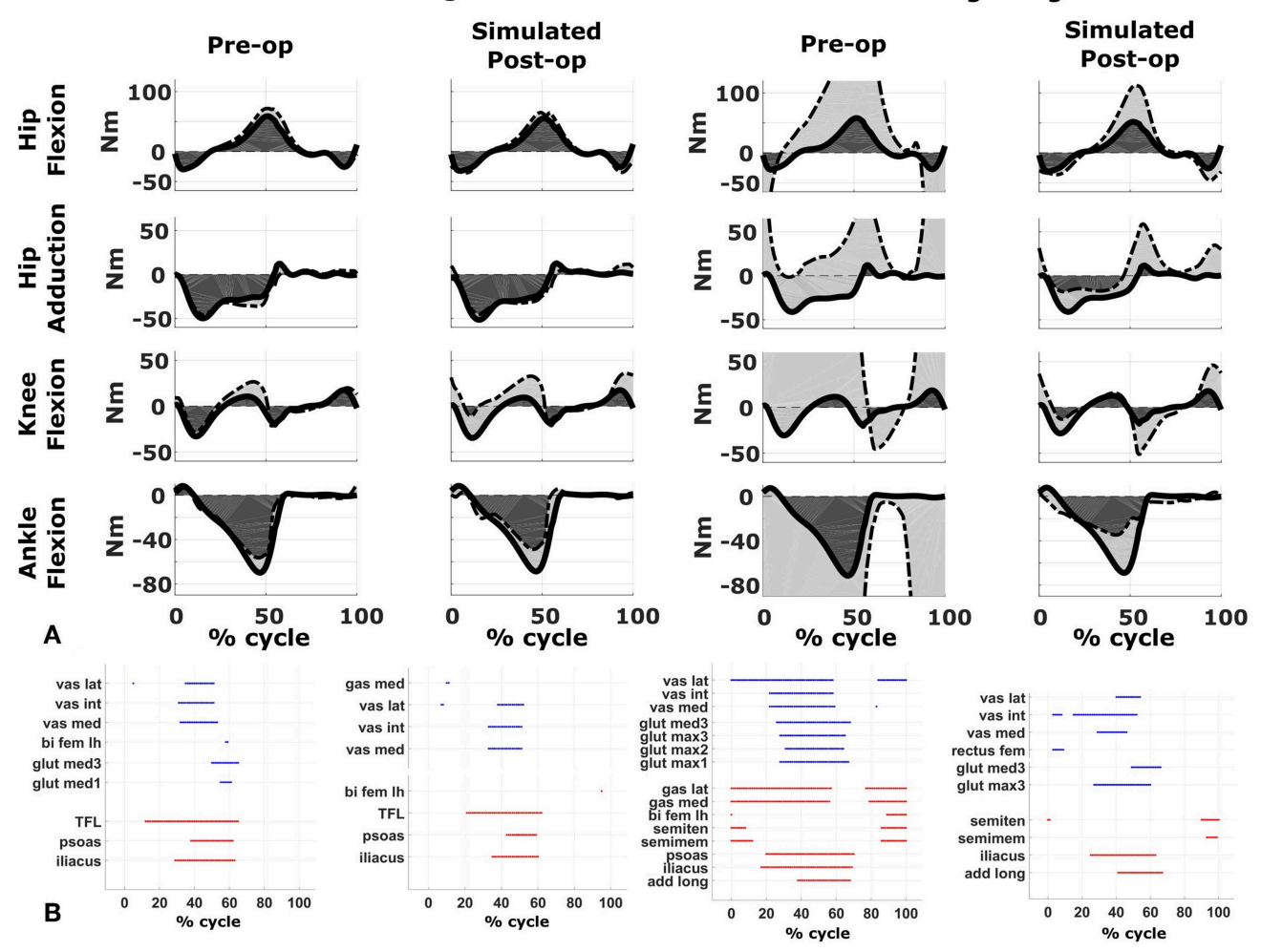

FIGURE 3 | Capability gap and report on muscle operating lengths. (A) Capability gap computed for the two representative patients, before and after the virtual surgery. The continuous black lines represent the joint moments required for the model to reach the desired kinematics [i.e., typically developed (TD) gait cycle]. Dotted black lines represent the moment exerted by the model. The light gray patches represent the capability gap. (B) Information about the operating conditions of the muscles. Blue dots represent the time instants in which muscles are active at short lengths (activation $>0.25$ and normalized fiber length smaller than 0.6 ), red dots the intervals in which muscles are stretched, and exert an excessive passive force ( $>0.5$ times the maximum isometric force). 


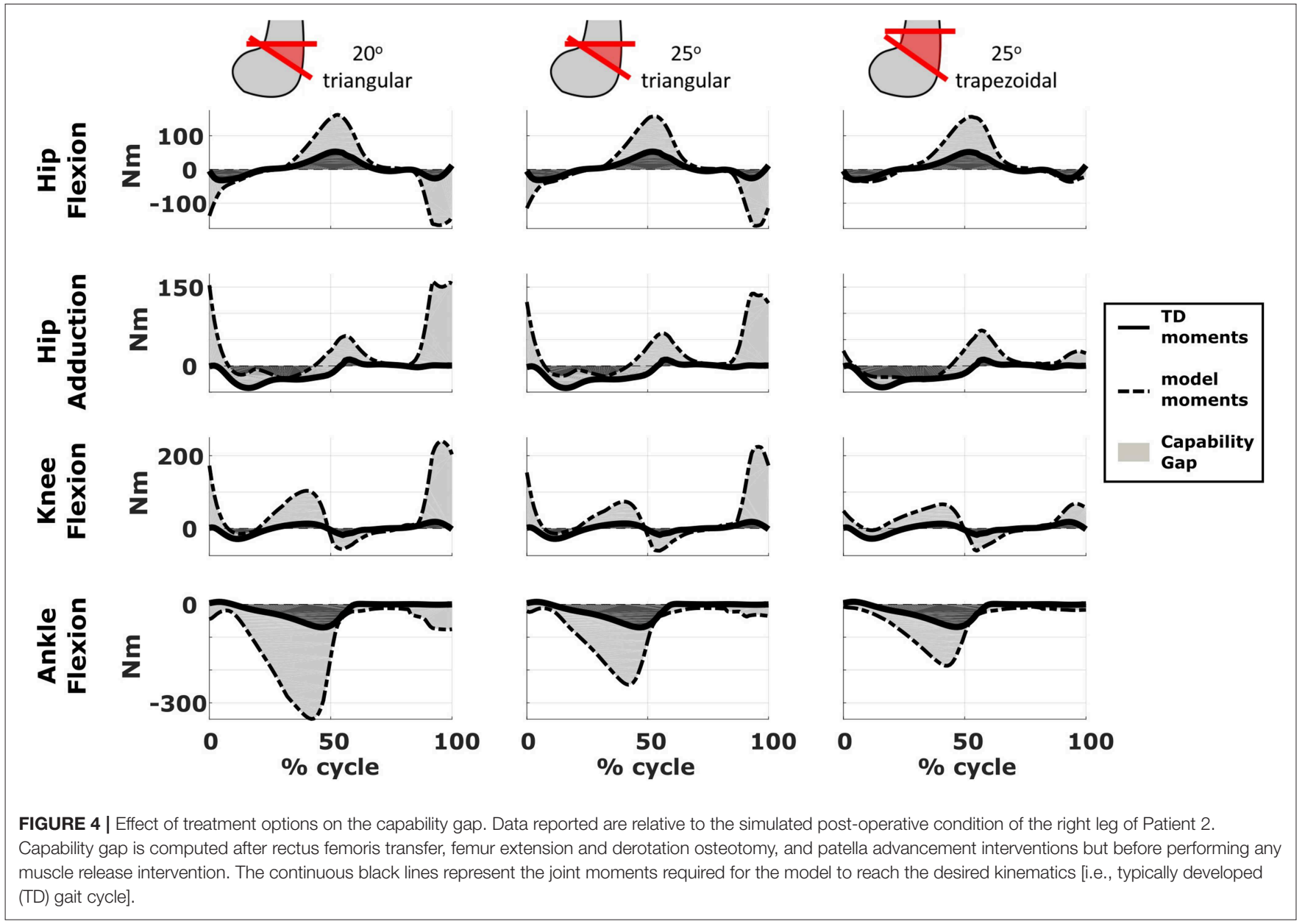

\section{RESULTS}

\section{Patient 1}

The patient had near normal range of motion at the hip and ankle, but a bilateral knee extension deficit in both limbs, bilateral spasticity in most muscles, including rectus femoris, good strength in most muscles bilaterally but slightly lower strength in hip and knee extensors, as well as hip abductors, with overall good selectivity (Table 1). The pre-operative gait analysis (Figure 6A) indicates bilateral excessive knee flexion and ankle dorsiflexion, with incomplete hip extension at the end of stance. The right side hip presents excessive hip adduction.

Synergy analysis revealed that three synergies were sufficient to describe the pre-operative EMG signals in both legs. For comparison, previous work of the group found that, during walking, $57 \%$ of $\mathrm{TD}$ children use four synergies, whereas the remainder of the subjects uses three. Therefore, to take into account muscles for which no EMG were collected, four synergies were used for the CG computation.

An important CG was found bilaterally at the level of the knees and to a lesser extent hip adduction (Figures 3A, 6B). The simulated interventions were able to reduce the calculated CG, especially at the knees, but also when averaged across all the joints. However, the effect on the CG at the level of the other joints was more variable. In particular, the left hip flexion and ankle dorsiflexion CG showed an increase after the surgery. The post-operative gait analysis shows that knee extension was restored successfully, whereas bilateral hip flexion increased after surgery. Ankle dorsiflexion was restored bilaterally, however, right ankle plantarflexion was still lacking.

The tuning of the muscle parameters was a necessary step to perform the aforementioned analyses. After applying the bony deformities to the model, most of the muscles would have operated at excessive values of $\tilde{l}$ (Figure 7). Therefore, this model would have been unable to generate the required joint moments due to excessive passive forces generation, introducing excessive muscle activations to compensate these and resulting in high residual torques. For Patient 1 , residual torques were as high as $45 \mathrm{Nm}$ for the knee joint. Nevertheless, parameter tuning was able to bring the residual torque values below $1 \mathrm{Nm}$ and to produce muscle activations closer to the measured EMG signals (Figure 7).

Within the framework, it is possible to evaluate the isolated effect of motor control deficit, by in- or excluding the motor control model when computing the post-operative CG. In Figure 5, the CG computed in both conditions is presented. 


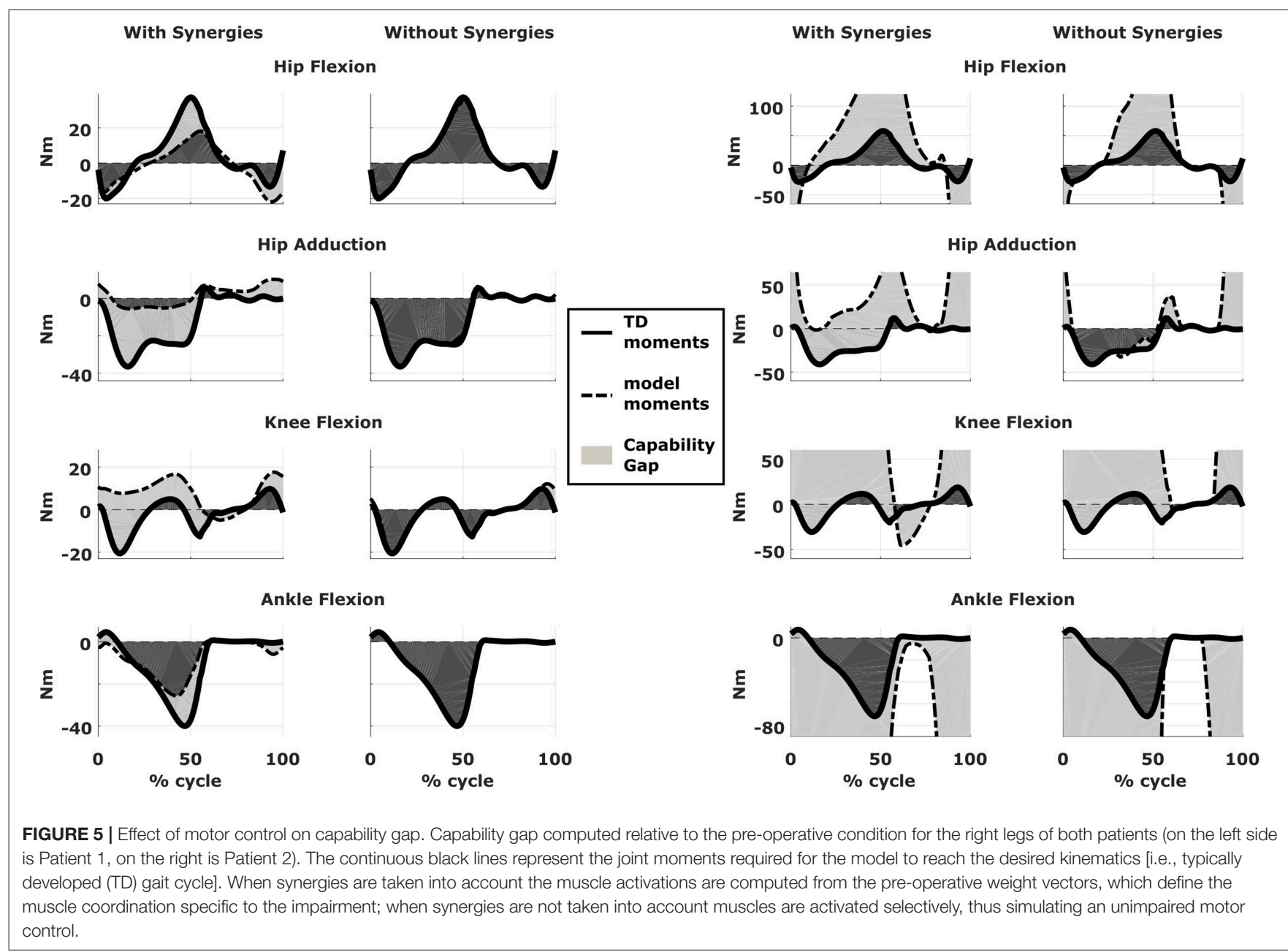

For this patient, the CG gap is almost zero when the synergy constraints on muscle activations are not considered. The impaired motor control has thus a major contribution to the CG for this patient.

\section{Patient 2}

The patient's right side was more involved in terms of passive ROM, spasticity and muscle weakness (Table 1). More specific, hip adduction was slightly decreased whereas knee extension and dorsiflexion were more severely restricted in the right limb. Spasticity was present overall, although more pronounced in the distal compared to the proximal muscles. Overall, the patient had good muscle strength, with slightly lower values for the right proximal muscles and hip abductors and for the left hip extensors and plantarflexor muscles. The pre-operative gait analysis revealed bilateral increased knee flexion and hip adduction. Ankle dorsiflexion was increased on the left side, whereas on the right side a reversed second rocker was present. A reversed second rocker is defined by dorsiflexion during loading response and the first half of mid-stance followed by plantarflexion, whereas during normal gait, second rocker is characterized by plantarflexion followed by dorsiflexion.
Insufficient hip extension in terminal stance was present at the left hip.

Synergy analysis revealed that four and three synergies could explain the pre-operative EMG signals for the left and right leg, respectively, thus leading to the use of five and four synergies in the CG computations. The CG in the preoperative condition reflected the reduced range of motion of the right leg. CG was higher for the right then for the left leg with muscles gastrocnemii, hamstrings, iliacus and psoas being excessively stretched (Figure 3B), leading to a large contribution of their passive forces to the CG. In addition, the comparison of the CG computed with and without the inclusion of the motor control (Figure 5) supports the interpretation that the vast majority of the CG is due to the aberrant musculoskeletal geometry and muscle properties, and not motor control.

The simulated treatment had a very different impact on the CG for the two legs. For the right leg, surgery massively reduced the CG. The simulated extension and derotation osteotomy in isolation reduced the CG generated by the hamstrings at the hip and knee at the beginning and end of the gait cycle. The use of a trapezoidal wedge, most commonly used 


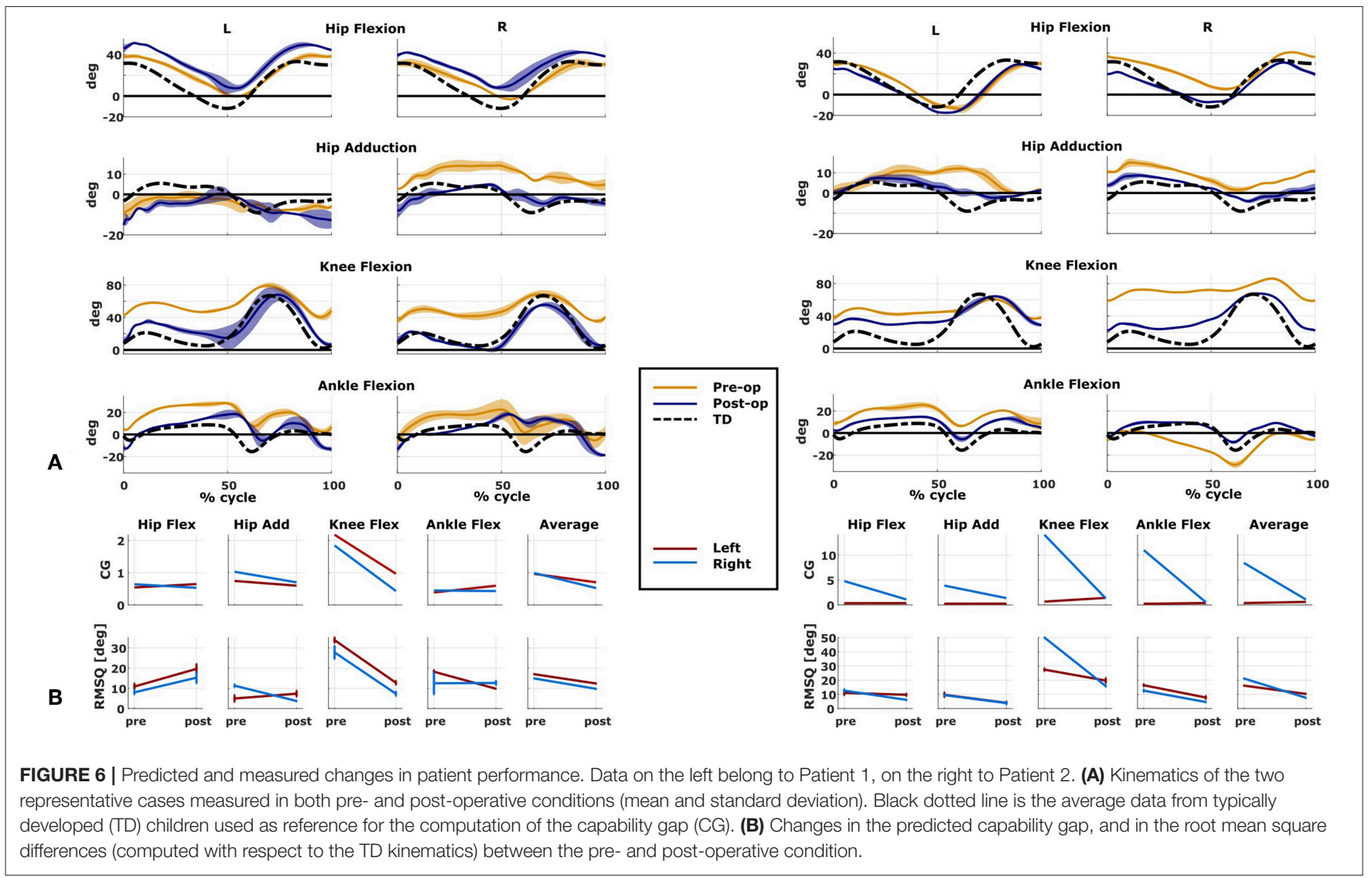

to correct large extension deficits, reduced the CG most by reducing excessive stretch in the muscles (Figure 4). The effect of the extension osteotomy was significant, but even after this treatment a large CG was present. This CG was most elevated in the stance phase for the ankle and knee joint and around toe-off for the hip joint. The muscle report indicated that the gastrocnemii, iliacus and psoas were markedly stretched during these intervals (Figure 3B). These muscles were targeted by the release procedure. The inclusion of these procedures within the framework further reduced the predicted CG as shown in Figure 3A. On the other hand, the pre-operative CG of the left leg was smaller and our framework predicted a slight increase in the CG after the intervention (Figures 3A, 6B).The post-operative gait analysis confirms the positive outcome, with a bilateral marked improvement in the observed kinematics (Figure 6).

\section{DISCUSSION}

We introduced a novel and promising musculoskeletal modeling and simulation-based framework to assist clinicians in the treatment selection process to improve gait function in patients with CP. The salient feature of this framework is a comprehensive personalization of the models comprising subject-specific musculoskeletal geometry and muscle parameters as well as motor control. Furthermore, we introduced a GUI to simulate different orthopedic interventions and interactively modify the musculoskeletal models. As a result, the effect of several candidate orthopedic interventions on the gait performance, evaluated in terms of the patient's capability gap, can be evaluated. In comparison with a number of other studies that aimed to predict the outcome of orthopedic treatments (Hicks et al., 2011; Schwartz et al., 2016; Galarraga et al., 2017), our method differs by the fact that the predictions within our framework are not based on statistical methods. This allows the user to select different treatments or combinations thereof and to evaluate their combined or isolated effects.

In comparison with studies applying forward predictive simulations (Fox et al., 2009; Mansouri et al., 2016), our framework offers the possibility to include a variety of interventions and to fine-tune their parameters: it is for instance possible to combine a muscle transfer or patella advancement surgery with a femoral extension derotation osteotomy and to specify the amount of bony correction. A recently published paper (Lee et al., 2019) proposes a similar framework in which it is possible to predict the post-operative gait after an orthopedic surgery. However, several differences with respect to our work are worth noticing. The model of the motor control used by Lee et al. is obtained via a trajectory mimicking policy, which does not take into account the patient's coordination strategy, whereas we include this feature using EMG based muscle synergies. 

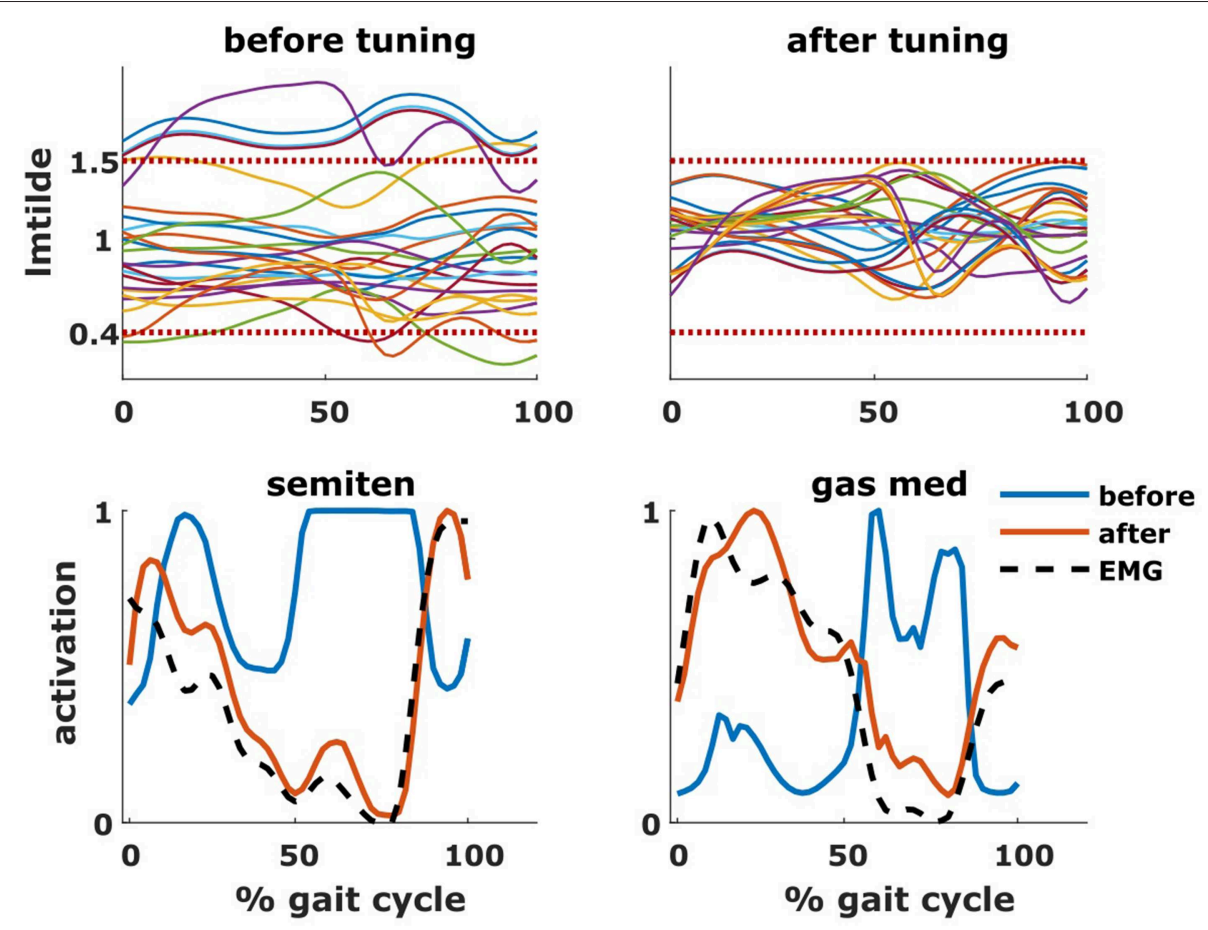

FIGURE 7 | Effect of tuning of muscle parameters. On the top row are plotted the values of the normalized muscle fiber lengths of all the tuned muscles throughout the gait cycle. The two horizontal dotted lines represent the constraints imposed during the optimization process. On the bottom row are plotted the activations of two representative muscles (semitendinosus and gastrocnemius medialis), before and after the optimization, as well as the experimental EMG signal.

While in Lee's work subject-specific muscle-tendon parameters are not included, we proposed a tuning based on detailed pre-operative information. In addition, there are differences in the proposed sets of interventions. Both studies implemented derotational osteotomies and muscle transfers, but Lee et al. included muscle-tendon lengthening while we included patella advancement and femoral extension osteotomy surgeries based on the interventions that are commonly used in CP treatment.

We demonstrated the potential of our framework based on two case studies. Indeed, we found a good agreement between the evolution of the predicted motor performance measured with the CG and the actual evolution of the patient kinematics. Using our framework, we were able to highlight the importance of taking into account the specific neurological and musculoskeletal impairments of the patients with CP when assessing gait dysfunction during the planning of an orthopedic intervention.

In Patient 1, virtual simulations of procedures correcting the bony deformities were able to reduce the CG. This agrees with the general trend of improved gait kinematics measured during the post-operative gait analysis. The CG computed without the inclusion of the motor control was almost negligible. Therefore, a hypothetical patient with the same musculoskeletal geometry and properties, but able to activate his/her muscles selectively, could be able to achieve a normal gait pattern. For this patient, the impaired motor control plays thus a major role in determining the altered gait pattern. It is interesting to note that, despite being mainly due to the impaired motor control, the CG of the patient is sensitive to the orthopedic intervention, suggesting an interaction between the motor control and musculoskeletal condition of the patient. In other words, motor control impairments might limit the compensation strategies that are available to patients with CP to counteract musculoskeletal deformities. In contrast, excluding the muscle synergies from the calculation of the CG for Patient 2 had little effect. This indicates that the abnormal muscle parameters, specifically the shortness of several muscles, are the main contributors to the altered gait pattern. This is evident in the muscle report, which indicates that many muscles, including gastrocnemius and psoas, operating at excessive lengths when the CG is high (Figure 3B). These findings support the need for additional muscular interventions on the right side, more specific the gastrocnemius and psoas release. The insights in the two case studies provided by our SimCP framework suggest that the underlying causes for the gait deviations might be very different in different patients, even when they present with similar gait patterns. The constraints imposed by the motor control and the musculoskeletal system should hence be taken into account during the clinical decision process.

Furthermore, we demonstrated that the implementation of a specific orthopedic intervention, more specific the choice of cutting planes and derotation magnitudes, might have a big influence on post-operative gait performance. By creating several models corresponding to different feasible variations in the surgical technique, it is possible to evaluate which variation 
has the highest potential to reduce the functional impairment of the patient. For example, increasing the angle of the bone wedge reduced the $C G$ in Patient 2, whereas a trapezoid wedge reduced the CG even more. In the future, we plan to develop an optimization based procedure to automatically identify the combination of surgical parameters that minimizes the predicted CG.

The presented framework has still several limitations that will be addressed in future studies. First, there is a need for validation of the model prediction. Although we showed the potential of the framework here, many more cases are needed to demonstrate that the CG is a valid measure of gait performance. To this aim, we will compare the computed change in CG with the measured change in gait kinematics induced by the treatment in a large population of CP children. In addition, the different subcomponents of the framework need further validation. For example, we plan to use MRI-images collected post-operative to validate our implementation of the surgical interventions. Second, all analyses are based on static optimization with musculotendon units having rigid tendons. This approach was chosen to reduce computational time and for ease of implementation. We are currently developing a dynamic optimization implementation that takes into account muscle dynamics enabling the inclusion of a model of muscle spasticity (Falisse et al., 2018). These developments might further improve prediction accuracy. Third, the CG does not predict how the patient will walk in terms of the kinematics. The CG does not describe how a patient will move after treatment but how difficult it would be for him/her to achieve a normal gait pattern. In other words, the CG does not provide any insight in possible kinematic compensation strategies. However, the CG has the advantage of being fast and easy to compute, requiring only a few seconds per trial, and thus enabling the comparison of multiple treatment options. In the near future, we plan to include predictive simulations of gait kinematics in our framework building on the workflow for personalized modeling that we presented here.

A beta version of the developed GUI is freely available (https:// simtk.org/projects/simcp), which will enable the biomechanical community to create post-operative models in an easy way and therefore foster future research related to orthopedic interventions and pathological gait. Furthermore, the GUI as well as the concept of the CG can be applied to different populations (e.g., stroke) and research questions (e.g., strength training).

\section{REFERENCES}

Afschrift, M., De Groote, F., De Schutter, J., and Jonkers, I. (2014). The effect of muscle weakness on the capability gap during gross motor function: a simulation study supporting design criteria for exoskeletons of the lower limb. Biomed. Eng. Online 13:111. doi: 10.1186/1475-925X-13-111

Allen, J. L., and Neptune, R. R. (2012). Three-dimensional modular control of human walking. J. Biomech. 45, 2157-2163. doi: 10.1016/j.jbiomech.2012.05.037

Arnold, A. S., Liu, M. Q., Schwartz, M. H., Ounpuu, S., and Delp, S. L. (2006a). The role of estimating muscle-tendon lengths and velocities of the hamstrings in the evaluation and treatment of crouch gait. Gait Posture 23, 273-281. doi: 10.1016/j.gaitpost.2005.03.003
To summarize, we conceptualized and developed a simulation-based framework that relies on highly personalized patient-specific models, including a description of the musculoskeletal geometry, the muscle parameters and the motor control. This framework is designed to assist clinicians in selecting the most promising treatment option for an individual patient solely based on pre-operative data. It is our aspiration that this in silico-informed clinical decision making framework will increase the number of positive treatment outcomes in ambulatory children with CP.

\section{DATA AVAILABILITY}

The datasets generated for this study are available on request to the corresponding author.

\section{ETHICS STATEMENT}

This study was carried out in accordance with the recommendations of the local ethical committee [Commissie Medische Ethiek KU Leuven (Medical Ethics Committee UZ $\mathrm{KU}$ Leuven/Research)]. In accordance with the Declaration of Helsinki, written informed consent was obtained of the participants' parents prior to the experiment. The participants parents supervised the measurement session. The protocol was approved by the Medical Ethics Committee UZ KU Leuven/Research.

\section{AUTHOR CONTRIBUTIONS}

LP, IJ, FD, KD, and $\mathrm{AH}$ contributed conception and design of the study. EP collected the data. AV and GM provided clinical insights for the methods development. LP, HK, AF, MW, SV, and $\mathrm{HH}$ contributed in developing the methods. LP, IJ, and FD wrote sections of the manuscript. All authors contributed to manuscript revision, read, and approved the submitted version.

\section{FUNDING}

Project was funded with IWT-TBM Grant Number: 140184. HK was funded by a H2020-MSCA individual fellowship (796120). AF was funded by the Research Foundation Flanders (FWO), Ph.D. grant 1 S35416N.

Arnold, A. S., Liu, M. Q., Schwartz, M. H., Ounpuu, S., Dias, L. S., and Delp, S. L. (2006b). Do the hamstrings operate at increased muscle-tendon lengths and velocities after surgical lengthening? J. Biomech. 39, 1498-1506. doi: 10.1016/j.jbiomech.2005.03.026

Baker, R., McGinley, J. L., Schwartz, M. H., Beynon, S., Rozumalski, A., Graham, H. K., et al. (2009). The gait profile score and movement analysis profile. Gait Posture 30, 265-269. doi: 10.1016/j.gaitpost.2009.05.020

Bosmans, L., Jansen, K., Wesseling, M., Molenaers, G., Scheys, L., and Jonkers, I. (2016). The role of altered proximal femoral geometry in impaired pelvis stability and hip control during CP gait: a simulation study. Gait Posture 44, 61-67. doi: 10.1016/j.gaitpost.2015.11.010

Bosmans, L., Wesseling, M., Desloovere, K., Molenaers, G., Scheys, L., and Jonkers, I. (2014). Hip contact force in presence of aberrant bone geometry 
during normal and pathological gait. J. Orthop. Res. 32, 1406-1415. doi: $10.1002 /$ jor. 22698

Cheung, V. C., d'Avella, A., and Bizzi, E. (2009). Adjustments of motor pattern for load compensation via modulated activations of muscle synergies during natural behaviors. J. Neurophysiol. 101, 1235-1257. doi: 10.1152/jn.01387.2007

Colver, A., Fairhurst, C., and Pharoah, P. O. D. (2014). Cerebral palsy. Lancet 383, 1240-1249. doi: 10.1016/S0140-6736(13)61835-8

De Groote, F., Jonkers, I., and Duysens, J. (2014). Task constraints and minimization of muscle effort result in a small number of muscle synergies during gait. Front. Comput. Neurosci. 8:115. doi: 10.3389/fncom.2014.00115

De Groote, F., Kinney, A. L., Rao, A. V., and Fregly, B. J. (2016). Evaluation of direct collocation optimal control problem formulations. Ann. Biomed. Eng. 44, 2922-2936. doi: 10.1007/s10439-016-1591-9

De Groote, F., Van Campen, A., Jonkers, I., and De Schutter, J. (2010). Sensitivity of dynamic simulations of gait and dynamometer experiments to hill muscle model parameters of knee flexors and extensors. J. Biomech. 43, 1876-1883. doi: 10.1016/j.jbiomech.2010.03.022

Delp, S. L., and Zajac, F. E. (1992). Force- and moment-generating capacity of lower-extremity muscles before and after tendon lengthening. Clin. Orthop. Relat. Res. 284, 247-259. doi: 10.1097/00003086-199211000-00035

Desloovere, K., Molenaers, G., Feys, H., Huenaerts, C., Callewaert, B., and Van de Walle, P. (2006). Do dynamic and static clinical measurements correlate with gait analysis parameters in children with cerebral palsy? Gait Posture 24, 302-313. doi: 10.1016/j.gaitpost.2005.10.008

Fairhurst, C. (2012). Cerebral palsy: the whys and hows. Arch. Dis. Child. Educ. Pract. Ed. 97, 122-131. doi: 10.1136/edpract-2011-300593

Falisse, A., Bar-On, L., Desloovere, K., Jonkers, I., De Groote, F. (2018). A spasticity model based on feedback from muscle force explains muscle activity during passive stretches and gait in children with cerebral palsy. PLoS ONE 13:e0208811. doi: 10.1371/journal.pone.0208811

Falisse, A., Van Rossom, S., Jonkers, I., and De Groote, F. (2017). EMGdriven optimal estimation of subject-SPECIFIC hill model muscle-tendon parameters of the knee joint actuators. IEEE Trans. Biomed. Eng. 64, 2253-2262. doi: 10.1109/TBME.2016.2630009

Filho, M. C., Yoshida, R., Carvalho Wda, S., Stein, H. E., and Novo, N. F. (2008). Are the recommendations from three-dimensional gait analysis associated with better postoperative outcomes in patients with cerebral palsy? Gait Posture 28, 316-322. doi: 10.1016/j.gaitpost.2008.01.013

Fitoussi, F., and Bachy, M. (2015). Tendon lengthening and transfer. Orthop. Traumatol. Surg. Res. 101, S149-S157. doi: 10.1016/j.otsr.2014.07.033

Fox, M. D., Reinbolt, J. A., Ounpuu, S., and Delp, S. L. (2009). Mechanisms of improved knee flexion after rectus femoris transfer surgery. J. Biomech. 42, 614-619. doi: 10.1016/j.jbiomech.2008.12.007

Galarraga, C. O. A., Vigneron, V., Dorizzi, B., Khouri, N., and Desailly, E. (2017). Predicting postoperative gait in cerebral palsy. Gait Posture 52, 45-51. doi: 10.1016/j.gaitpost.2016.11.012

Graham, H. K., Rosenbaum, P., Paneth, N., Dan, B., Lin, J., Damiano, D. L., et al. (2016). Cerebral palsy. Nat. Rev. Dis. Prim. 2:15082. doi: 10.1038/nrdp.2015.82

Halilaj, E., Rajagopal, A., Fiterau, M., Hicks, J. L., Hastie, T. J., and Delp, S. L. (2018). Machine learning in human movement biomechanics: best practices, common pitfalls, and new opportunities. J. Biomech. 81, 1-11. doi: 10.1016/j.jbiomech.2018.09.009

Hanna, S. E., Rosenbaum, P. L., Bartlett, D. J., Palisano, R. J., Walter, S. D., Avery, L., et al. (2009). Stability and decline in gross motor function among children and youth with cerebral palsy aged 2 to 21 years. Dev. Med. Child Neurol. 51, 295-302. doi: 10.1111/j.1469-8749.2008.03196.x

Hersh, L. A., Sun, J. Q., Richards, J. G., and Miller, F. (2002). The prediction of post-operative gait patterns using neural networks. Gait Posture 5:151. doi: 10.1016/S0966-6362(97)83371-1

Hicks, J. L., Delp, S. L., and Schwartz, M. H. (2011). Can biomechanical variables predict improvement in crouch gait? Gait Posture 34, 197-201. doi: 10.1016/j.gaitpost.2011.04.009

Hicks, J. L., Uchida, T. K., Seth, A., Rajagopal, A., and Delp, S. L. (2015). Is my model good enough? Best practices for verification and validation of musculoskeletal models and simulations of movement. J. Biomech. Eng. 137:020905. doi: 10.1115/1.4029304

Kainz, H., Goudriaan, M., Falisse, A., Huenaerts, C., Desloovere, K., De Groote, F., et al. (2018). The influence of maximum isometric muscle force scaling on estimated muscle forces from musculoskeletal models of children with cerebral palsy. Gait Posture 65, 213-220. doi: 10.1016/j.gaitpost.2018.07.172

Kalkman, B. M., Bar-On, L., Cenni, F., Maganaris, C. N., Bass, A., Holmes, G., et al. (2018). Muscle and tendon lengthening behaviour of the medial gastrocnemius during ankle joint rotation in children with cerebral palsy. Exp. Physiol. 103, 1367-1376. doi: 10.1113/EP087053

Kim, Y., Bulea, T. C., and Damiano, D. L. (2018). Children with cerebral palsy have greater stride-to-stride variability of muscle synergies during gait than typically developing children: implications for motor control complexity. Neurorehabil. Neural Repair 32, 834-844. doi: 10.1177/1545968318796333

Kruse, A., Schranz, C., Svehlik, M., and Tilp, M. (2017). Mechanical muscle and tendon properties of the plantar flexors are altered even in highly functional children with spastic cerebral palsy. Clin. Biomech. 50, 139-144. doi: 10.1016/j.clinbiomech.2017.10.019

Lee, D. D., and Seung, H. S. (1999). Learning the parts of objects by non-negative matrix factorization. Nature 401, 788-791. doi: 10.1038/44565

Lee, S., Lee, K., Park, M., and Lee, J. (2019). Scalable muscle-actuated human simulation and control. ACM Trans. Graph. 37:73.

Lenhart, R. L., Smith, C. R., Schwartz, M. H., Novacheck, T. F., and Thelen, D. G. (2017). The effect of distal femoral extension osteotomy on muscle lengths after surgery. J. Child. Orthop. 11, 472-478. doi: 10.1302/1863-2548.11.170087

Mansouri, M., Clark, A. E., Seth, A., and Reinbolt, J. A. (2016). Rectus femoris transfer surgery affects balance recovery in children with cerebral palsy: a computer simulation study. Gait Posture 43, 24-30. doi: 10.1016/j.gaitpost.2015.08.016

Meyer, A. J., Eskinazi, I., Jackson, J. N., Rao, A. V., Patten, C., and Fregly, B. J. (2016). Muscle synergies facilitate computational prediction of subject-specific walking motions. Front. Bioeng. Biotechnol. 4:77. doi: 10.3389/fbioe.2016.00077

Modenese, L., Ceseracciu, E., Reggiani, M., and Lloyd, D. G. (2016). Estimation of musculotendon parameters for scaled and subject specific musculoskeletal models using an optimization technique. J. Biomech. 49, 141-148. doi: 10.1016/j.jbiomech.2015.11.006

Molenaers, G., Desloovere, K., De Cat, J., Jonkers, I., De Borre, L., Pauwels, P., et al. (2001). Single event multilevel botulinum toxin type A treatment and surgery: similarities and differences. Eur. J. Neurol. 8, 88-97. doi: 10.1046/j.1468-1331.2001.00041.x

Moon, S. J., Choi, Y., Chung, C. Y., Sung, K. H., Cho, B. C., Chung, M. K., et al. (2017). Normative values of physical examinations commonly used for cerebral palsy. Yonsei Med. J. 58, 1170-1176. doi: 10.3349/ymj.2017.58.6.1170

Morrison, T. M., Pathmanathan, P., Adwan, M., and Margerrison, E. (2018). Advancing regulatory science with computational modeling for medical devices at the FDA's office of science and engineering laboratories. Front. Med. 5:241. doi: 10.3389/fmed.2018.00241

Narayanan, U. G. (2012). Management of children with ambulatory cerebral palsy: an evidence-based review. J. Pediatr. Orthop. 32 (suppl. 2), S172-S181. doi: 10.1097/BPO.0b013e31825eb2a6

Nieuwenhuys, A., Papageorgiou, E., Pataky, T., De Laet, T., Molenaers, G., and Desloovere, K. (2016). Literature review and comparison of two statistical methods to evaluate the effect of botulinum toxin treatment on gait in children with cerebral palsy. PLoS ONE 11:e0152697. doi: 10.1371/journal.pone.0152697

Niiler, T. A., Richards, J. G., and Miller, F. (2007). Concurrent surgeries are a factor in predicting success of rectus transfer outcomes. Gait Posture 26, 76-81. doi: 10.1016/j.gaitpost.2006.07.014

Opheim, A., Jahnsen, R., Olsson, E., and Stanghelle, J. K. (2009). Walking function, pain, and fatigue in adults with cerebral palsy: a 7-year follow-up study. Dev. Med. Child Neurol. 51, 381-388. doi: 10.1111/j.1469-8749.2008.03250.x

Patikas, D., Wolf, S. I., Schuster, W., Armbrust, P., Dreher, T., and Döderlein, L. (2007). Electromyographic patterns in children with cerebral palsy: do they change after surgery? Gait Posture 26, 362-371. doi: 10.1016/j.gaitpost.2006.10.012

Pitto, L., Kaat, D., Guy, M., Catherine, H., Friedl, D. G., and Ilse, J. (2018) O 109 - Post-treatment muscle coordination patterns during gait are highly similar to pre-treatment ones in CP children. Gait Posture 65, 228-229. doi: 10.1016/j.gaitpost.2018.06.144

Prado, L. G., Makarenko, I., Andresen, C., Krüger, M., Opitz, C. A., and Linke, W. A. (2005). Isoform diversity of giant proteins in relation to passive and active contractile properties of rabbit skeletal muscles. J. Gen. Physiol. 126, 461-480. doi: 10.1085/jgp.200509364 
Reinbolt, J. A., Fox, M. D., Schwartz, M. H., and Delp, S. L. (2009). Predicting outcomes of rectus femoris transfer surgery. Gait Posture 30, 100-105. doi: 10.1016/j.gaitpost.2009.03.008

Sartori, M., Fernandez, J. W., Modenese, L., Carty, C. P., Barber, L. A., Oberhofer, K., et al. (2017). Toward modeling locomotion using electromyographyinformed 3D models: application to cerebral palsy. Wiley Interdiscip. Rev. Syst. Biol. Med. 9:e1368. doi: 10.1002/wsbm.1368

Sartori, M., Gizzi, L., Lloyd, D. G., and Farina, D. (2013). A musculoskeletal model of human locomotion driven by a low dimensional set of impulsive excitation primitives. Front. Comput. Neurosci. 7:79. doi: 10.3389/fncom.2013. 00079

Scheys, L., Desloovere, K., Spaepen, A., Suetens, P., and Jonkers, I. (2011b). Calculating gait kinematics using MR-based kinematic models. Gait Posture 33, 158-164. doi: 10.1016/j.gaitpost.2010.11.003

Scheys, L., Desloovere, K., Suetens, P., and Jonkers, I. (2011a). Level of subjectspecific detail in musculoskeletal models affects hip moment arm length calculation during gait in pediatric subjects with increased femoral anteversion. J. Biomech. 44, 1346-1353. doi: 10.1016/j.jbiomech.2011.01.001

Scheys, L., Van Campenhout, A., Spaepen, A., Suetens, P., and Jonkers, I. (2008). Personalized MR-based musculoskeletal models compared to rescaled generic models in the presence of increased femoral anteversion: effect on hip moment arm lengths. Gait Posture 28, 358-365. doi: 10.1016/j.gaitpost.2008. 05.002

Schwartz, M. H., Rozumalski, A., and Steele, K. M. (2016). Dynamic motor control is associated with treatment outcomes for children with cerebral palsy. Dev. Med. Child Neurol. 58, 1139-1145. doi: 10.1111/dmcn.13126

Schwartz, M. H., Rozumalski, A., Truong, W., and Novacheck, T. F. (2013). Predicting the outcome of intramuscular psoas lengthening in children with cerebral palsy using preoperative gait data and the random forest algorithm. Gait Posture 37, 473-479. doi: 10.1016/j.gaitpost.2012. 08.016

Sherman, M. A., Seth, A., and Delp, S. L. (2014). "What is a moment arm? Calculating muscle effectiveness in biomechanical models using generalized coordinates," in Proceedings of the ASME 2013 International Design Engineering Technical Conferences \& Computers and Information in Engineering Conference. doi: 10.1115/DETC2013-13633
Steele, K. M., Rozumalski, A., and Schwartz, M. H. (2015). Muscle synergies and complexity of neuromuscular control. Dev. Med. Child Neurol. 57, 1176-1182. doi: $10.1111 / \mathrm{dmcn} .12826$

Strobl, W., Theologis, T., Brunner, R., Kocer, S., Viehweger, E., Pascual-Pascual, I., et al. (2015). Best clinical practice in botulinum toxin treatment for children with cerebral palsy. Toxins. 7, 1629-1648. doi: 10.3390/toxins7051629

Theis, N., Mohagheghi, A. A., and Korff, T. (2016). Mechanical and material properties of the plantar fl exor muscles and Achilles tendon in children with spastic cerebral palsy and typically developing children. J. Biomech. 49, 3004-3008. doi: 10.1016/j.jbiomech.2016.07.020

Van Campen, A., Pipeleers, G., De Groote, F., Jonkers, I., and De Schutter, J. (2014). A new method for estimating subject-specific muscle-tendon parameters of the knee joint actuators: a simulation study. Int. J. Numer. Method. Biomed. Eng. 30, 969-987. doi: 10.1002/cnm.2639

Van Der Krogt, M. M., Bar-On, L., Kindt, T., Desloovere, K., and Harlaar, J. (2016). Neuro-musculoskeletal simulation of instrumented contracture and spasticity assessment in children with cerebral palsy. J. Neuroeng. Rehabil. 13:64. doi: 10.1186/s12984-016-0170-5

Wren, T. A.L, Kalisvaart, M. M., Ghatan, C. E., Rethlefsen, S. A., Hara, R., Sheng, M., et al. (2009). Effects of preoperative gait analysis on costs and amount of surgery. J. Pediatr. Orthop. 29, 558-563. doi: 10.1097/BPO.0b013e3181b2f8c,2

Zajac, F. E. (1989). Muscle and tendon: properties, models, scaling, and application to biomechanics and motor control. Crit. Rev. Biomed. Eng. 17, 359-411.

Conflict of Interest Statement: The authors declare that the research was conducted in the absence of any commercial or financial relationships that could be construed as a potential conflict of interest.

Copyright (c) 2019 Pitto, Kainz, Falisse, Wesseling, Van Rossom, Hoang, Papageorgiou, Hallemans, Desloovere, Molenaers, Van Campenhout, De Groote and Jonkers. This is an open-access article distributed under the terms of the Creative Commons Attribution License (CC BY). The use, distribution or reproduction in other forums is permitted, provided the original author(s) and the copyright owner(s) are credited and that the original publication in this journal is cited, in accordance with accepted academic practice. No use, distribution or reproduction is permitted which does not comply with these terms. 